\title{
APOE-TOMM40 in the Pharmacogenomics of Dementia
}

\author{
Ramón Cacabelos ${ }^{1 *}$, Dmitry Goldgaber ${ }^{2}$, Alexander Vostrov², Hideyuki Matsuki ${ }^{2}$, Clara Torrellas ${ }^{1}$, Dolores Corzo ${ }^{1}$, Juan Carlos Carril ${ }^{1}$ and \\ Allen D Roses
}

${ }^{1}$ Chair of Genomic Medicine, Camilo José Cela University, Madrid; EuroEspes Biomedical Research Center, Institute for Medical Science and Genomic Medicine,

Corunna, Spain

${ }^{2}$ Department of Psychiatry and Behavioral Science, Stony Brook University, New York, USA

${ }^{3}$ Zinfandel Pharmaceuticals, Inc., Durham, NC; Duke University Medical Center, Department of Neurology, Durham, NC, USA

\begin{abstract}
Polymorphic variants present in the APOE-TOMM40 region (19q13.2) are implicated in Alzheimer's disease $(A D)$ and have been shown to modify disease risk, age at onset of symptoms, and the therapeutic response to conventional drugs. We have investigated the structure of the APOE-TOMM40 region and the influence of APOE and TOMM40 poly T variants (rs10524523) in the therapeutic response to a multifactorial treatment in 920 Spanish patients with $\mathrm{AD}$. The frequencies of TOMM40 poly T genotypes were: $18.37 \% \mathrm{~S} / \mathrm{S}, 7.83 \% \mathrm{~S} / \mathrm{L}, 38.80 \% \mathrm{~S} / \mathrm{VL}$, $1.52 \% \mathrm{~L} / \mathrm{L}, 7.17 \% \mathrm{~L} / \mathrm{VL}$, and $26.31 \% \mathrm{VL} / \mathrm{VL}$. The most frequent APOE-TOMM40 associations were as follows: $82 \%$ APOE-3/3 with S/S, $63 \%$ with S/VL, and $40 \%$ with VL/VL; $90 \%$ APOE-3/4 with S/L, 57\% with L/VL, and $43 \%$ with VL/VL; and $100 \%$ APOE-4/4 with L/L. Globally, the response rate was about $59 \%$, with no difference between females and males. APOE- 4 carriers were found to be the worst responders (45-56\%) whereas APOE-3/3 carriers were the best responders $(70 \%)$ for a transient period of cognitive improvement $(<12 \mathrm{~m})$. Among TOMM40 variants, S/S carriers were the best responders $(70 \%)$, followed by S/VL $(61 \%)$, VL/VL $(57 \%)$, and L/VL carriers $(51 \%)$. The worst responders were those patients harboring the L/L genotype (35\%). Therefore, specific APOE-TOMM40 poly $\mathrm{T}$ variants exert a powerful influence on the therapeutic response to conventional treatments in AD.
\end{abstract}

Keywords: Alzheimer's disease; APOE; Multifactorial treatment; Pharmacogenomics; Therapeutic Response Rate; TOMM40
Abbreviations:
A2BP1: Ataxin-2 Binding Protein 1; ABAT: 4-aminobutyrate aminotransferase; ABCA7: ATP-binding cassette, sub-family A (ABC1), member 7; ABCB1: ATP-binding cassette, sub-family B (MDR/TAP), member 1; ABCs: ATP- binding cassette family; $\mathrm{ABO}$ : $\mathrm{ABO}$ blood group (transferase A, alpha 1-3-N-acetylgalactosaminyltransferase; transferase B, alpha 1-3-galactosyltransferase); ACE: Angiotensin I Converting Enzyme; AD: Alzheimer's Disease; AGT: Angiotensinogen; APOC1: Apolipoprotein C-I; APOC2: Apolipoprotein C-II; APOC4: Apolipoprotein C-IV; APOE: Apolipoprotein E; APOs: Apolipoproteins; APP: Amyloid beta (A4) Precursor Protein; BIN1: Bridging Integrator 1; CD2AP: CD2-associated protein; $\mathrm{CDH} 2$ : Cadherin 2, type 1; CLU: Clusterin; CNVs: Copy Number Variations; CR1: Complement Component (3b/4b) Receptor 1; CRMP1: Collapsin Response Mediator Protein 1; CRP: C-reactive protein; CVD: Cardiovascular Disease; CYP2D6: Cytochrome P450, family 2, subfamily D, polypeptide 6; CYPs: Cytochrome P450 family; DMRT1: Doublesex and mab-3 related transcription factor 1; EO-FAD: Early- onset Familial Alzheimer's Disease; EPHA5: EPH receptor A5; EPHA6: EPH receptor A6; ERMP1: Endoplasmic Reticulum metallopeptidase 1; GWAS: Genome-wide Association Studies; HMGCR: 3-hydroxy- 3-methylglutaryl-CoA reductase; ILs: Interleukins; L: Long; LOAD: Late Onset Alzheimer's Disease; MAPT: Microtubule-associated protein tau; MS4A4E: Membrane-spanning 4-domains, subfamily A, member 4E ; MS4A6A: Membrane-spanning 4-domains, subfamily A, member 6A; MTHFR: Methylenetetrahydrofolate Reductase (NAD $(\mathrm{P})$ $\mathrm{H})$; NATs: N-acetyltransferase family; NFT: Neurofibrillary Tangles; NOS: Nitric Oxide Synthase; PICALM: Phosphatidylinositol binding clathrin assembly protein; PRNP: Prion protein; PSEN1: Presenilin 1; PSEN2: Presenilin 2; S: Short; SLCs: Solute Carrier Family; SNPs: Single-nucleotide Polymorphisms; TOMM40: Translocase of Outer Mitochondrial Membrane 40 homolog (yeast); UGTs: UDP glucuronosyltransferase family; VL: Very Long; VLDLR: Very Low Density Lipoprotein Receptor

\section{Introduction}

The genes involved in the pharmacogenomic response to drugs in Alzheimer's disease (AD) fall into five major categories: (i) genes associated with $\mathrm{AD}$ pathogenesis and neurodegeneration (APP, PSEN1, PSEN2, MAPT, PRNP, APOE and others); (ii) genes associated with the mechanism of action of drugs (enzymes, receptors, transmitters, messengers); (iii) genes associated with drug metabolism (phase I $(C Y P s)$ and phase II reactions (UGTs, NATs); (iv) genes associated with drug transporters $(A B C s, S L C s)$; and (v) pleiotropic genes involved in multifaceted cascades and metabolic reactions (APOs, ILs, MTHFR, $A C E, A G T, N O S$, etc) [1-4]. The genetic and epigenetic defects identified so far in $\mathrm{AD}$ include Mendelian mutations, susceptibility single-nucleotide polymorphisms (SNPs), mitochondrial DNA (mtDNA) mutations, and epigenetic changes. Mendelian mutations affect genes directly linked to $\mathrm{AD}$, including mutations in the amyloid beta precursor protein $(A P P)$ gene $(21 \mathrm{q} 21)(A D 1)$, mutations in the presenilin 1 (PSEN1) gene (14q24.3)(AD3), and mutations in the presenilin 2 (PSEN2) gene (1q31-q42) (AD4) [5-10]. PSEN1 and PSEN2 are important determinants of $\gamma$-secretase activity responsible for proteolytic cleavage of APP and NOTCH receptor proteins. Mendelian mutations are very rare in $\mathrm{AD}$ (1:1000). Mutations in exons 16 and 17 of the APP gene appear with a frequency of $0.30 \%$ and $0.78 \%$, respectively, in AD patients. Likewise, PSEN1, PSEN2, and

*Corresponding author: Prof. Dr. Ramón Cacabelos, Chair of Genomic Medicine, Camilo José Cela University, Castillo de Alarcón, 49 Urb. Villafranca del CastilloVillanueva de la Cañada 28692-Madrid, Spain, Tel: +34 91815 3131; E-mail: rcacabelos@ucjc.edu

Received April 24, 2014; Accepted June 27, 2014; Published July 04, 2014

Citation: Cacabelos R, Goldgaber D, Vostrov A, Matsuki H, Torrellas C, et al. (2014) APOE-TOMM40 in the Pharmacogenomics of Dementia. J Pharmacogenomics Pharmacoproteomics 5: 135. doi:10.4172/2153-0645.1000135

Copyright: (c) 2014 Cacabelos R, et al. This is an open-access article distributed under the terms of the Creative Commons Attribution License, which permits unrestricted use, distribution, and reproduction in any medium, provided the original author and source are credited. 
microtubule-associated protein Tau (MAPT) (17q21.1) mutations are present in less than $2 \%$ of the cases. In the Alzgene database [11] there are over 600 genes potentially associated with $\mathrm{AD}$, of which the top ten are APOE (19q13.2), BIN1 (2q14), CLU (8p21-p12), ABCA7 (19p13.3), CR1 (1q32), PICALM (11q14), MS4A6A (11q12.1), CD33 (19q13.3), MS4A4E (11q12.2), and CD2AP (6p12) [6]. Potentially defective genes associated with $\mathrm{AD}$ represent about $1.39 \%(35,252.69 \mathrm{~Kb})$ of the human genome, which is integrated by 36,505 genes $(3,095,677.41$ $\mathrm{Kb})$. The highest number of AD-related defective genes concentrate on chromosomes 10 (5.41\%; $7337.83 \mathrm{~Kb}), 21(4.76 \% ; 2,289,15 \mathrm{~Kb}), 7$ $(1.62 \% ; 2,584.26 \mathrm{~Kb}), 2(1.56 \% ; 3,799.67 \mathrm{~Kb}), 19$ (1.45\%; $854.54 \mathrm{~Kb}), 9$ $(1.42 \% ; 2,010.62 \mathrm{~Kb}), 15$ (1.23\%; 1,264.4 Kb), 17 (1.19\%; $970.16 \mathrm{~Kb}), 12$ $(1.17 \% ; 1,559.9 \mathrm{~Kb})$, and $6(1.15 \% ; 1,968.22 \mathrm{~Kb})$ [3]. Ten novel private pathogenic copy number variations (CNVs) in 10 early-onset familial Alzheimer's disease (EO-FAD) families overlapping a set of genes (A2BP1, ABAT, CDH2, CRMP1, DMRT1, EPHA5, EPHA6, ERMP1, $E V C, E V C 2, F L J 35024$ and $V L D L R)$ have also been identified [3].

Multiple polymorphic risk variants can increase neuronal vulnerability to premature death. Among these susceptibility genes, the apolipoprotein $\mathrm{E}$ (APOE) gene (19q13.2) (AD2) is the most prevalent as a risk factor for $\mathrm{AD}$, especially in those subjects harboring the $A P O E$ 4 allele $[12,13]$, whereas carriers of the $A P O E-2$ allele are prone to longevity [14] and might be protected against dementia [15-17].

$A P O E$ is the prototypical paradigm of a pleiotropic gene with multifaceted activities in physiological and pathological conditions $[5,18]$. APOE is consistently associated with the amyloid plaque marker for $\mathrm{AD}$. APOE-4 may influence AD pathology by interacting with APP metabolism and $\mathrm{A} \beta$ accumulation, enhancing hyperphosphorylation of tau protein and neurofibrillary tangles (NFT) formation, reducing choline acetyltransferase activity, increasing oxidative processes, modifying inflammation-related neuroimmunotrophic activity and glial activation, altering lipid metabolism, lipid transport and membrane biosynthesis in sprouting and synaptic remodeling, and inducing neuronal apoptosis [5,18-21]. In addition, multiple studies over the past two decades have demonstrated that APOE variants may affect the therapeutic response to anti-dementia drugs [20-30].

The TOMM40 locus is located adjacent to and in linkage disequilibrium with APOE on 19q13.2. A poly T repeat in an intronic polymorphism (rs10524523) (intron 6) in the TOMM40 gene, which encodes an outer mitochondrial membrane translocase involved in the transport of amyloid- $\beta$ and other proteins into mitochondria, has been implicated in AD [31-44], and APOE-TOMM40 genotypes have been shown to modify disease risk and age at onset of symptoms [32-37,45], although the latter assumption needs replication due to contradictory results [36,46-49]. A fixed-effect meta-analysis approach showed that rs4420638 at the TOMM40/APOE/APOC1 gene locus is associated with longevity $[50,51]$. Two independent associations with cognitive decline were found among European-Americans in the 19q13.32 region (rs769449, APOE intron; and rs115881343, TOMM40 intron); rs769449 was also associated with cognitive decline among African-Americans, but rs115881343 was not [52]. The APOE-TOMM40 genomic region is associated with cognitive aging [53] and with pathological cognitive decline [54].

Linnertz et al. [55] investigated the genomic region spanning the TOMM40 and APOE genes, to determine whether intronic poly $\mathrm{T}$ (rs10524523) within this region affects expression of the APOE and TOMM40 genes in the brain of patients with late-onset Alzheimer's disease (LOAD). The expression of both genes was significantly increased with disease. Mean expression of APOE and TOMM40 mRNA levels was higher in VL homozygotes compared with S homozygotes in the temporal and occipital cortexes from normal and LOAD cases. The 523 VL poly $T$ resulted in significantly higher expression than the $\mathrm{S}$ poly $\mathrm{T}$. These results suggest that the 523 locus may contribute to LOAD susceptibility by modulating the expression of TOMM40 and/or APOE transcription [55]. Recent studies also suggest that the TOMM40 gene rs 10524523 ("523”) variable length poly T repeat polymorphism is associated to a certain extent with similar AD phenotypes as those reported for APOE, such as brain white matter changes [56,57] or different biomarkers [58-61]. In addition, the TOMM40 rs2075650 $\mathrm{G}$ allele may be a risk factor for the development of depression [62] and sporadic inclusion body myositis [63]. Different markers at the 19q13-q13.2 chromosomal region, including the rs2075650 and rs157590 (TOMM40), rs1064725 (APOC1), and rs429358 and rs7412 (APOE) SNPs also show association with primary progressive aphasia and the behavioural variant frontotemporal dementia [64].

The TOMM40/APOE/APOC1 loci have been associated with C-reactive protein (CRP), a heritable biomarker of systemic inflammation and a predictor of cardiovascular disease (CVD) [65] Genome-wide association studies (GWAS) have identified LDLcholesterol-associated loci near HMGCR, ABO and TOMM40 [66], and also an association of TOMM40 with blood lipid levels $[67,68]$ and body mass index [69]. Genetic variants in TOMM40/APOEC1-C2-C4 genes have also been found to be associated with multiple cardiovascular-related traits [70-72].

In the present study we have investigated the structure of the APOETOMM40 region in Spanish patients with dementia, and the influence of polymorphic variants in this genomic segment on the therapeutic response to a multifactorial treatment adapted to the pathogenic profile of the patients. The main aims of the study were: (i) structural analysis of the APOE-TOMM40 region (distribution and frequency of major genotypes, with special emphasis on TOMM40 poly T variants) in the Spanish population with dementia; and (ii) APOE- and TOMM40 poly T1/T2-related therapeutic response to a multifactorial therapy in AD.

\section{Materials and Methods}

\section{Patients and treatment}

In this retrospective study, selected Spanish patients with $\mathrm{AD}$ (DSM-IV/NINCDS-ADRDA criteria), treated at the EuroEspes Biomedical Research Center (period 1992-2011)(N=920, age: 71.11 \pm 9.09 years, range: $41-96$ years; 556 females, age: $71.14 \pm 9.07$ years, range: $43-96$ years; and 364 males, age: $71.06 \pm 9.13$ years, range: $41-$ 94 years), received for one year a multifactorial therapy integrated by CDP-choline (500 mg/day, p.o.) (choline donor and intermediate metabolite in DNA synthesis and repair), Piracetam $(1600 \mathrm{mg} / \mathrm{day}$, p.o.) (nootropic drug), Sardilipin (E-SAR-94010) (250 mg, t.i.d.) (nutraceutical with lipid-lowering effects and anti-atherosclerotic properties, Patent ID: P9602566) [3,24,73], and Animon Complex (2 capsules/day) (a nutraceutical compound integrated by a purified extract of Chenopodium quinoa $(250 \mathrm{mg})$, ferrous sulphate $(38.1 \mathrm{mg}$ equivalent to $14 \mathrm{mg}$ of iron), folic acid $(200 \mu \mathrm{g})$, and vitamin $\mathrm{B}_{12}(1$ $\mu \mathrm{g})$ per capsule (RGS: $26.06671 / \mathrm{C})$ ). Patients with chronic deficiency of iron $(<35 \mu \mathrm{g} / \mathrm{mL})(4.45 \%)$, folic acid $(<3 \mathrm{ng} / \mathrm{mL})(5.43 \%)$ or vitamin $\mathrm{B}_{12}(<170 \mathrm{pg} / \mathrm{mL})(4.13 \%)$ received an additional supplementation of iron $(80 \mathrm{mg} /$ day $)$, folic acid $(5 \mathrm{mg} /$ day $)$ and $B$ complex vitamins $\left(B_{1}\right.$, $15 \mathrm{mg} /$ day; $\mathrm{B}_{2}, 15 \mathrm{mg} /$ day; $\mathrm{B}_{6}, 10 \mathrm{mg} /$ day; $\mathrm{B}_{12}, 10 \mu \mathrm{g} /$ day; nicotinamide, $50 \mathrm{mg} /$ day), respectively, to maintain stable levels of serum iron (50$150 \mu \mathrm{g} / \mathrm{mL})$, folic acid $(5-20 \mathrm{ng} / \mathrm{mL})$ and vitamin $B_{12}$ levels (500-1000 $\mathrm{pg} / \mathrm{mL}$ ) in order to avoid the negative influence of these metabolic 
Citation: Cacabelos R, Goldgaber D, Vostrov A, Matsuki H, Torrellas C, et al. (2014) APOE-TOMM40 in the Pharmacogenomics of Dementia. J Pharmacogenomics Pharmacoproteomics 5: 135. doi:10.4172/2153-0645.1000135

factors on cognition [27,28]. Patients with hypertension $(>150 / 85$ $\mathrm{mmHg}$ (28.04\%) received enalapril (5-20 $\mathrm{mg} /$ day, p.o.); patients with hypercholesterolemia $(>220 \mathrm{mg} / \mathrm{dL})(43.48 \%)$ received atorvastatin (10-20 mg/day); patients with diabetes (glucose $>105 \mathrm{mg} / \mathrm{dL})(24.24 \%)$ received metformin $(850-1700 \mathrm{mg} /$ day, p.o. $)$; and patients $(<3 \%)$ with other ailments (e.g. hypothyroidism, hyperuricemia, etc.) received the appropriate treatment according to their medical condition. Psychotropic drugs (antidepressants, neuroleptics, hypnotics, sedatives) were avoided, and less than $5 \%$ of the patients required a transient treatment with benzodiazepines for short periods of time. Blood pressure, psychometric assessment (Mini-Mental State Examination, MMSE; Alzheimer's Disease Assessment Scale, ADAS Hamilton Rating Scale-Depression, HAM-D; Hamilton Rating ScaleAnxiety, HAM-A), and blood parameters Table 1 were evaluated prior to treatment (baseline) and after 1, 3, 6, 9, and 12 months of treatment. All patients and/or their legal representatives gave informed consent for genotyping and clinical assessment.

\section{APOE genotyping}

Genomic DNA was extracted from EDTA-treated blood samples

\begin{tabular}{|c|c|c|c|c|}
\hline $\begin{array}{c}\text { Parameter } \\
\text { (normal range) }\end{array}$ & Total & Females & Males & Differences \\
\hline $\mathrm{N}$ & 920 & 556 & 364 & \\
\hline $\begin{array}{l}\text { Age (years) } \\
\text { Range }\end{array}$ & $\begin{array}{c}71.11 \pm 9.09 \\
41-96\end{array}$ & $\begin{array}{c}71.14 \pm 9.07 \\
43-96\end{array}$ & $\begin{array}{c}71.06 \pm 9.13 \\
41-94\end{array}$ & $P=0.74$ \\
\hline $\begin{array}{c}\text { Systolic Blood Pressure (SBP) } \\
(\mathrm{mm} \mathrm{Hg}) \\
(120-160)\end{array}$ & $\begin{array}{c}140.61 \pm 20.64 \\
<120: 122(13.26 \%) \\
>160: 188(20.43 \%)\end{array}$ & $\begin{array}{c}140.10 \pm 20.66 \\
74(13.31 \%) \\
105(18.88 \%)\end{array}$ & $\begin{array}{c}141.38 \pm 20.62 \\
48(13.18 \%) \\
83(22.80 \%)\end{array}$ & $\begin{array}{c}P=0.41 \\
P\left(X^{2}\right)=0.92 \\
P\left(X^{2}\right)=0.15\end{array}$ \\
\hline $\begin{array}{c}\text { Diastolic Blood Pressure (DBP) } \\
(\mathrm{mm} \mathrm{Hg}) \\
(70-85)\end{array}$ & $\begin{array}{c}79.49 \pm 11.89 \\
<70: 71(7.72 \%) \\
>85: 258(28.04 \%)\end{array}$ & $\begin{array}{c}79.52 \pm 11.70 \\
40(7.19 \%) \\
142(25.54 \%)\end{array}$ & $\begin{array}{c}79.64 \pm 11.62 \\
31(8.51 \%) \\
116(31.87 \%)\end{array}$ & $\begin{array}{c}P=0.71 \\
P\left(X^{2}\right)=0.52 \\
P\left(X^{2}\right)=0.04\end{array}$ \\
\hline $\begin{array}{l}\text { Pulse (bpm) } \\
\quad(60-100)\end{array}$ & $\begin{array}{c}67.93 \pm 12.23 \\
<60: 224(24.34 \%) \\
>100: 15(1.63 \%)\end{array}$ & $\begin{array}{c}68.74 \pm 11.65 \\
117(21.04 \%) \\
8(1.44 \%)\end{array}$ & $\begin{array}{c}66.69 \pm 12.96 \\
107(29.39 \%) \\
7(1.92 \%)\end{array}$ & $\begin{array}{c}P=0.001 \\
P\left(X^{2}\right)=0.005 \\
P\left(X^{2}\right)=0.60\end{array}$ \\
\hline Weight $(\mathrm{Kg})$ & $69.70 \pm 12.93$ & $65.39 \pm 11.76$ & $76.54 \pm 11.71$ & $P<0.001$ \\
\hline Height (m) & $1.58 \pm 0.09$ & $1.53 \pm 0.06$ & $1.66 \pm 0.06$ & $P<0.001$ \\
\hline $\begin{array}{c}\text { Body mass index }(\mathrm{BMI})\left(\mathrm{Kg} / \mathrm{m}^{2}\right) \\
\text { Underweight }(15-18.5) \\
\text { Normal weight }(18.5-25) \\
\text { Overweight }(25-30) \\
\text { Obese Class I (moderate)(30-35) } \\
\text { Obese Class II (severe)(35-40) } \\
\text { Obese Class III (very severe)(>40) }\end{array}$ & $\begin{array}{c}27.88 \pm 4.45 \\
10(1.08 \%) \\
309(33.59 \%) \\
382(41.52 \%) \\
162(17.61 \%) \\
49(5.33 \%) \\
8(0.87 \%)\end{array}$ & $\begin{array}{c}28.01 \pm 4.95 \\
8(1.44 \%) \\
185(33.27 \%) \\
213(38.31 \%) \\
102(18.35 \%) \\
41(7.37 \%) \\
7(1.26 \%)\end{array}$ & $\begin{array}{c}27.68 \pm 3.49 \\
2(0.55 \%) \\
124(34.07 \%) \\
169(46.43 \%) \\
60(16.48 \%) \\
8(2.20 \%) \\
1(0.27 \%)\end{array}$ & $\begin{array}{c}P=0.72 \\
P\left(X^{2}\right)=0.33 \\
P\left(X^{2}\right)=0.83 \\
P\left(X^{2}\right)=0.01 \\
P\left(X^{2}\right)=0.48 \\
P\left(X^{2}\right)=0.001 \\
P\left(X^{2}\right)=0.15\end{array}$ \\
\hline $\begin{array}{c}\text { Glucose }(\mathrm{Glc})(\mathrm{mg} / \mathrm{dL}) \\
(70-105)\end{array}$ & $\begin{array}{c}\text { 101.77 } \pm 27.36 \\
<70: 6(0.65 \%) \\
>105: 223(24.24 \%)\end{array}$ & $\begin{array}{c}98.74 \pm 25.03 \\
3(0.54 \%) \\
103(18.52 \%)\end{array}$ & $\begin{array}{c}106.37 \pm 30.02 \\
3(0.82 \%) \\
120(32.97 \%)\end{array}$ & $\begin{array}{c}P<0.001 \\
P\left(X^{2}\right)=0.68 \\
P\left(X^{2}\right)=0.0005\end{array}$ \\
\hline $\begin{array}{c}\text { Cholesterol (Cho) }(\mathrm{mg} / \mathrm{dL}) \\
(140-220)\end{array}$ & $\begin{array}{c}221.17 \pm 47.75 \\
<140: 31(3.37 \%) \\
>220: 400(43.48 \%)\end{array}$ & $\begin{array}{c}231.69 \pm 46.28 \\
9(1.62 \%) \\
336(60.43 \%)\end{array}$ & $\begin{array}{c}205.12 \pm 45.48 \\
22(6.04 \%) \\
64(17.58 \%)\end{array}$ & $\begin{array}{c}P<0.001 \\
P\left(X^{2}\right)=0.0005 \\
P\left(X^{2}\right)=0.0005\end{array}$ \\
\hline $\begin{array}{c}\text { HDL-Cholesterol (mg/dL) } \\
(35-75)\end{array}$ & $\begin{array}{c}53.88 \pm 14.43 \\
<35: 59(6.41 \%) \\
>75: 86(9.35 \%)\end{array}$ & $\begin{array}{c}58.17 \pm 14.45 \\
15(2.67 \%) \\
72(12.95 \%)\end{array}$ & $\begin{array}{c}47.39 \pm 11.76 \\
44(12.09 \%) \\
14(3.85 \%)\end{array}$ & $\begin{array}{c}P<0.001 \\
P\left(X^{2}\right)=0.0005 \\
P\left(X^{2}\right)=0.0005\end{array}$ \\
\hline $\begin{array}{l}\text { LDL-Cholesterol (mg/dL) } \\
\quad(80-160)\end{array}$ & $\begin{array}{c}144.37 \pm 41.62 \\
<80: 44(4.78 \%) \\
>160: 318(34.56 \%)\end{array}$ & $\begin{array}{c}150.47 \pm 41.49 \\
16(2.88 \%) \\
219(39.39 \%)\end{array}$ & $\begin{array}{c}135.16 \pm 40.16 \\
28(7.69 \%) \\
99(27.20 \%)\end{array}$ & $\begin{array}{c}P<0.001 \\
P\left(X^{2}\right)=0.001 \\
P\left(X^{2}\right)=0.0005\end{array}$ \\
\hline $\begin{array}{c}\text { Triglycerides (TG) }(\mathrm{mg} / \mathrm{dL}) \\
(50-150)\end{array}$ & $\begin{array}{c}113.51 \pm 65.36 \\
<50: 49(5.33 \%) \\
>150: 180(19.56 \%)\end{array}$ & $\begin{array}{c}111.56 \pm 64.65 \\
32(5.75 \%) \\
97(17.45 \%)\end{array}$ & $\begin{array}{c}116.50 \pm 66.41 \\
17(4.67 \%) \\
83(22.80 \%)\end{array}$ & $\begin{array}{c}P=0.38 \\
P\left(X^{2}\right)=0.54 \\
P\left(X^{2}\right)=0.05\end{array}$ \\
\hline $\begin{array}{c}\text { Urea (BUN) (mg/dL) } \\
(15-30)\end{array}$ & $\begin{array}{c}43.93 \pm 13.52 \\
>30: 825(89.67 \%)\end{array}$ & $\begin{array}{l}42.33 \pm 12.53 \\
466(83.81 \%)\end{array}$ & $\begin{array}{l}46.39 \pm 14.60 \\
359(98.62 \%)\end{array}$ & $\begin{array}{c}P<0.001 \\
P\left(X^{2}\right)=0.0005\end{array}$ \\
\hline $\begin{array}{c}\text { Creatinine }(\mathrm{Cr})(\mathrm{mg} / \mathrm{dL}) \\
(0.70-1.40)\end{array}$ & $\begin{array}{c}0.92 \pm 0.47 \\
<0.70: 129(14.02 \%) \\
>1.40: 56(6.08 \%)\end{array}$ & $\begin{array}{c}0.81 \pm 0.17 \\
123(22.12 \%) \\
18(3.24 \%)\end{array}$ & $\begin{array}{c}1.08 \pm 0.68 \\
6(0.82 \%) \\
38(10.44 \%)\end{array}$ & $\begin{array}{c}P<0.001 \\
P\left(X^{2}\right)=0.0005 \\
P\left(X^{2}\right)=0.0005\end{array}$ \\
\hline $\begin{array}{c}\text { Uric Acid (UA) (mg/dL) } \\
(3.4-7.0)\end{array}$ & $\begin{array}{c}4.27 \pm 1.43 \\
<3.4: 260(28.26 \%) \\
>7.0: 45(4.89 \%)\end{array}$ & $\begin{array}{c}3.81 \pm 1.29 \\
226(40.65 \%) \\
13(2.34 \%)\end{array}$ & $\begin{array}{l}4.97 \pm 1.35 \\
34(9.34 \%) \\
32(8.79 \%)\end{array}$ & $\begin{array}{c}P<0.001 \\
P\left(X^{2}\right)=0.0005 \\
P\left(X^{2}\right)=0.0005\end{array}$ \\
\hline $\begin{array}{c}\text { Total Protein }(\mathrm{T}-\mathrm{Pro})(\mathrm{g} / \mathrm{dL}) \\
(6.5-8.0)\end{array}$ & $\begin{array}{c}6.92 \pm 0.43 \\
<6.5: 123(13.37 \%) \\
>8.0: 8(0.87 \%)\end{array}$ & $\begin{array}{c}6.94 \pm 0.43 \\
68(12.23 \%) \\
5(0.90 \%)\end{array}$ & $\begin{array}{c}6.89 \pm 0.43 \\
55(15.11 \%) \\
3(0.82 \%)\end{array}$ & $\begin{array}{c}P=0.07 \\
P\left(X^{2}\right)=0.23 \\
P\left(X^{2}\right)=0.80\end{array}$ \\
\hline $\begin{array}{c}\text { Albumin (Alb) }(\mathrm{g} / \mathrm{dL}) \\
(3.5-5.0)\end{array}$ & $\begin{array}{c}4.28 \pm 0.29 \\
<3.5: 7(0.76 \%) \\
>5.0: 7(0.76 \%)\end{array}$ & $\begin{array}{c}4.28 \pm 0.29 \\
3(0.54 \%) \\
3(0.54 \%)\end{array}$ & $\begin{array}{c}4.28 \pm 0.29 \\
4(1.10 \%) \\
4(1.10 \%)\end{array}$ & $\begin{array}{c}P=0.92 \\
P\left(X^{2}\right)=0.44 \\
P\left(X^{2}\right)=0.44\end{array}$ \\
\hline $\begin{array}{c}\text { Calcium }(\mathrm{Ca})(\mathrm{mg} / \mathrm{dL}) \\
(8.1-10.4)\end{array}$ & $\begin{array}{c}9.21 \pm 0.44 \\
<8.1: 1(0.11 \%) \\
>10.4: 14(1.52 \%)\end{array}$ & $\begin{array}{c}9.21 \pm 0.45 \\
0(0 \%) \\
8(0.87 \%)\end{array}$ & $\begin{array}{c}9.18 \pm 0.42 \\
1(0.27 \%) \\
6(1.65 \%)\end{array}$ & $\begin{array}{c}P=0.48 \\
P\left(X^{2}\right)=0.39 \\
P\left(X^{2}\right)=0.80\end{array}$ \\
\hline $\begin{array}{c}\text { Phosphorus }(P)(\mathrm{mg} / \mathrm{dL}) \\
(2.5-5.0)\end{array}$ & $\begin{array}{c}3.37 \pm 0.54 \\
<2.5: 29(3.15 \%) \\
>5.0: 7(0.76 \%)\end{array}$ & $\begin{array}{c}3.45 \pm 0.52 \\
14(2.52 \%) \\
2(0.36 \%)\end{array}$ & $\begin{array}{c}3.25 \pm 0.54 \\
15(4.12 \%) \\
5(1.37 \%)\end{array}$ & $\begin{array}{c}P<0.001 \\
P\left(X^{2}\right)=0.18 \\
P\left(X^{2}\right)=0.12\end{array}$ \\
\hline
\end{tabular}


Citation: Cacabelos R, Goldgaber D, Vostrov A, Matsuki H, Torrellas C, et al. (2014) APOE-TOMM40 in the Pharmacogenomics of Dementia. J Pharmacogenomics Pharmacoproteomics 5: 135. doi:10.4172/2153-0645.1000135

Page 4 of 12

\begin{tabular}{|c|c|c|c|c|}
\hline $\begin{array}{l}\text { Aspartate Aminotransferase (GOT/ASAT) (IU/L) } \\
\qquad(10-40)\end{array}$ & $\begin{array}{c}22.58 \pm 20.14 \\
<10: 2(0.22 \%) \\
>40: 40(4.35 \%)\end{array}$ & $\begin{array}{c}22.64 \pm 23.24 \\
2(0.36 \%) \\
13(2.34 \%)\end{array}$ & $\begin{array}{c}22.49 \pm 14.20 \\
0(\%) \\
27(7.42 \%)\end{array}$ & $\begin{array}{c}P=0.30 \\
P\left(X^{2}\right)=0.52 \\
P\left(X^{2}\right)=0.0005\end{array}$ \\
\hline $\begin{array}{l}\text { Alanine Aminotransferase (GPT/ALAT) (IU/L) } \\
\qquad(9-43)\end{array}$ & $\begin{array}{c}23.80 \pm 22.60 \\
<9: 21(2.28 \%) \\
>43: 60(6.52 \%)\end{array}$ & $\begin{array}{c}22.07 \pm 21.13 \\
21(3.77 \%) \\
24(4.32 \%)\end{array}$ & $\begin{array}{c}26.46 \pm 24.47 \\
0(\%) \\
36(9.89 \%)\end{array}$ & $\begin{array}{c}P<0.001 \\
P\left(X^{2}\right)=0.0005 \\
P\left(X^{2}\right)=0.001\end{array}$ \\
\hline $\begin{array}{l}\text { Gamma-glutamyl transpeptidase (GGT) (IU/L) } \\
\qquad(11-50)\end{array}$ & $\begin{array}{c}30.76 \pm 41.97 \\
<11: 65(7.06 \%) \\
>50: 110(11.95 \%)\end{array}$ & $\begin{array}{c}27.51 \pm 41.01 \\
57(10.25 \%) \\
52(9.35 \%)\end{array}$ & $\begin{array}{c}35.69 \pm 42.99 \\
8(2.20 \%) \\
58(15.93 \%)\end{array}$ & $\begin{array}{c}P<0.001 \\
P\left(X^{2}\right)=0.0005 \\
P\left(X^{2}\right)=0.003\end{array}$ \\
\hline $\begin{array}{l}\text { Alkaline Phosphatase (ALP) (IU/L) } \\
\qquad(37-111)\end{array}$ & $\begin{array}{c}78.34 \pm 30.51 \\
<37: 12(1.30 \%) \\
>111: 130(14.13 \%)\end{array}$ & $\begin{array}{c}79.93 \pm 31.71 \\
8(1.44 \%) \\
79(14.21 \%)\end{array}$ & $\begin{array}{c}75.90 \pm 28.46 \\
4(1.10 \%) \\
51(14.01 \%)\end{array}$ & $\begin{array}{c}P=0.01 \\
P\left(X^{2}\right)=0.77 \\
P\left(X^{2}\right)=0.93\end{array}$ \\
\hline $\begin{array}{l}\text { Bilirubin }(\mathrm{BIL})(\mathrm{mg} / \mathrm{dL}) \\
\quad(0.20-1.00)\end{array}$ & $\begin{array}{c}0.74 \pm 0.35 \\
<0.20: 3(0.37 \%) \\
>1.00: 158(17.17 \%)\end{array}$ & $\begin{array}{c}0.69 \pm 0.30 \\
2(0.36 \%) \\
68(12.23 \%)\end{array}$ & $\begin{array}{c}0.82 \pm 0.42 \\
1(0.27 \%) \\
90(24.72 \%)\end{array}$ & $\begin{array}{c}P<0.001 \\
P\left(X^{2}\right)=0.82 \\
P\left(X^{2}\right)=0.0005\end{array}$ \\
\hline $\begin{array}{l}\text { Creatine Phosphokinase }(\mathrm{CPK})(\mathrm{IU} / \mathrm{L}) \\
(38-174)\end{array}$ & $\begin{aligned} & 85.41 \pm 59.51 \\
< & 38: 96(10.43 \%) \\
> & 174: 75(8.15 \%)\end{aligned}$ & $\begin{array}{c}82.51 \pm 57.92 \\
53(9.53 \%) \\
38(6.83 \%)\end{array}$ & $\begin{array}{c}89.80 \pm 61.67 \\
43(11.81 \%) \\
37(10.16 \%)\end{array}$ & $\begin{array}{c}P=0.20 \\
P\left(X^{2}\right)=0.27 \\
P\left(X^{2}\right)=0.08\end{array}$ \\
\hline $\begin{array}{l}\text { Lactate Dehydrogenase (LDH) (IU/L) } \\
(200-480)\end{array}$ & $\begin{array}{c}304.11 \pm 71.75 \\
<200: 16(1.74 \%) \\
>480: 30(3.26 \%)\end{array}$ & $\begin{array}{c}316.16 \pm 68.13 \\
5(0.90 \%) \\
9(1.62 \%)\end{array}$ & $\begin{array}{c}285.74 \pm 73.34 \\
11(3.02 \%) \\
21(5.77 \%)\end{array}$ & $\begin{array}{c}P<0.001 \\
P\left(X^{2}\right)=0.02 \\
P\left(X^{2}\right)=0.001\end{array}$ \\
\hline $\begin{array}{l}\mathrm{Na}^{+}(\mathrm{mEq} / \mathrm{L}) \\
(135-148)\end{array}$ & $\begin{array}{c}142.18 \pm 2.47 \\
<135: 7(0.76 \%) \\
>148: 15(1.63 \%)\end{array}$ & $\begin{array}{c}142.35 \pm 2.36 \\
3(0.54 \%) \\
7(1.26 \%)\end{array}$ & $\begin{array}{c}141.91 \pm 2.61 \\
4(1.10 \%) \\
8(2.20 \%)\end{array}$ & $\begin{array}{c}P=0.005 \\
P\left(X^{2}\right)=0.44 \\
P\left(X^{2}\right)=0.29\end{array}$ \\
\hline $\begin{array}{l}\mathrm{K}^{+}(\mathrm{mEq} / \mathrm{L}) \\
(3.5-5.3)\end{array}$ & $\begin{array}{c}4.35 \pm 0.37 \\
<3.5: 9(0.98 \%) \\
>5.3: 45(4.89 \%)\end{array}$ & $\begin{array}{c}4.29 \pm 0.36 \\
8(1.44 \%) \\
0(0 \%)\end{array}$ & $\begin{array}{c}4.44 \pm 0.37 \\
1(0.27 \%) \\
45(12.36 \%)\end{array}$ & $\begin{array}{c}P<0.001 \\
P\left(X^{2}\right)=0.09 \\
P\left(X^{2}\right)=0.0005\end{array}$ \\
\hline $\begin{array}{l}\mathrm{Cl}^{-}(\mathrm{mEq} / \mathrm{L}) \\
(98-107)\end{array}$ & $\begin{array}{c}104.18 \pm 2.54 \\
<98: 13(1.41 \%) \\
>107: 60(6.52 \%)\end{array}$ & $\begin{array}{c}104.36 \pm 2.48 \\
5(0.90 \%) \\
42(7.55 \%)\end{array}$ & $\begin{array}{c}103.90 \pm 2.62 \\
8(2.20 \%) \\
18(4.94 \%)\end{array}$ & $\begin{array}{c}P=0.01 \\
P\left(X^{2}\right)=0.15 \\
P\left(X^{2}\right)=0.13\end{array}$ \\
\hline $\begin{array}{l}\mathrm{Fe}^{2+}(\mu \mathrm{g} / \mathrm{dL}) \\
\quad(35-160)\end{array}$ & $\begin{array}{c}84.65 \pm 33.88 \\
<35: 41(4.45 \%) \\
>160: 15(1.63 \%)\end{array}$ & $\begin{array}{c}80.04 \pm 31.75 \\
28(5.03 \%) \\
8(1.44 \%)\end{array}$ & $\begin{array}{c}91.09 \pm 36.12 \\
13(3.57 \%) \\
7(1.92 \%)\end{array}$ & $\begin{array}{c}P<0.001 \\
P\left(X^{2}\right)=0.33 \\
P\left(X^{2}\right)=0.60\end{array}$ \\
\hline $\begin{array}{l}\text { Ferritin }(\mathrm{ng} / \mathrm{mL}) \\
(\mathrm{F}: 11-307) \\
(\mathrm{M}: 24-336)\end{array}$ & $\begin{array}{c}118.57 \pm 136.38 \\
<11: 26(2.83 \%) \\
>307: 44(4.78 \%)\end{array}$ & $\begin{array}{c}88.21 \pm 109.60 \\
<11: 26(4.67 \%) \\
>307: 11(55.21 \%)\end{array}$ & $\begin{array}{c}162.53 \pm 158.09 \\
<24: 23(6.32 \%) \\
>336: 33(9.06 \%)\end{array}$ & $\begin{array}{c}P<0.001 \\
P\left(X^{2}\right)=0.36 \\
P\left(X^{2}\right)=0.0005\end{array}$ \\
\hline $\begin{array}{l}\text { Folate }(\mathrm{ng} / \mathrm{mL}) \\
\qquad(>3.00)\end{array}$ & $\begin{aligned} & 7.01 \pm 3.88 \\
< & 3: 50(5.43 \%)\end{aligned}$ & $\begin{array}{l}7.12 \pm 3.79 \\
30(5.39 \%)\end{array}$ & $\begin{array}{l}6.84 \pm 4.01 \\
20(5.49 \%)\end{array}$ & $\begin{array}{c}P=0.06 \\
P\left(X^{2}\right)=0.94\end{array}$ \\
\hline $\begin{array}{c}\text { Vitamin } B_{12}(\mathrm{pg} / \mathrm{mL}) \\
(170-1000)\end{array}$ & $\begin{array}{l}509.21 \pm 314.45 \\
<170: 38(4.13 \%)\end{array}$ & $\begin{array}{c}507.48 \pm 312.21 \\
24(4.32 \%)\end{array}$ & $\begin{array}{c}511.84 \pm 318.23 \\
14(3.84 \%)\end{array}$ & $\begin{array}{c}P=0.92 \\
P\left(X^{2}\right)=0.74\end{array}$ \\
\hline $\begin{array}{l}\text { Thyroid-stimulating Hormone }(\mathrm{TSH})(\mu \mathrm{IU} / \mathrm{mL}) \\
\qquad(0.20-4.50)\end{array}$ & $\begin{array}{c}1.49 \pm 2.86 \\
<0.20: 33(3.58 \%) \\
>4.50: 18(1.95 \%)\end{array}$ & $\begin{array}{l}1.62 \pm 3.58 \\
24(4.32 \%) \\
13(2.34 \%)\end{array}$ & $\begin{array}{c}1.29 \pm 1.06 \\
9(2.47 \%) \\
5(1.37 \%)\end{array}$ & $\begin{array}{c}P=0.04 \\
P\left(X^{2}\right)=0.15 \\
P\left(X^{2}\right)=0.34\end{array}$ \\
\hline $\begin{array}{l}\text { Thyroxine }(\mathrm{T} 4)(\mathrm{ng} / \mathrm{mL}) \\
(0.54-1.40)\end{array}$ & $\begin{array}{c}0.94 \pm 0.35 \\
<0.54: 9(0.98 \%) \\
>1.40: 67(7.28 \%)\end{array}$ & $\begin{array}{c}0.93 \pm 0.23 \\
8(1.44 \%) \\
26(4.67 \%)\end{array}$ & $\begin{array}{c}0.94 \pm 0.48 \\
1(0.27 \%) \\
41(11.26 \%)\end{array}$ & $\begin{array}{c}P=0.41 \\
P\left(X^{2}\right)=0.09 \\
P\left(X^{2}\right)=0.0005\end{array}$ \\
\hline $\begin{array}{l}\text { Red Blood Cell Count (RBC) }\left(\times 10^{6} / \mu \mathrm{L}\right) \\
\qquad(3.80-5.50)\end{array}$ & $\begin{array}{c}4.59 \pm 0.45 \\
<3.80: 27(2.93 \%) \\
>5.50: 26(2.82 \%)\end{array}$ & $\begin{array}{c}4.47 \pm 0.39 \\
21(3.77 \%) \\
3(0.54 \%)\end{array}$ & $\begin{array}{c}4.78 \pm 0.48 \\
6(1.65 \%) \\
23(6.32 \%)\end{array}$ & $\begin{array}{c}P<0.001 \\
P\left(X^{2}\right)=0.07 \\
P\left(X^{2}\right)=0.0005\end{array}$ \\
\hline $\begin{array}{l}\text { Hematocrit }(\mathrm{HCT})(\%) \\
(40.0-50.0)\end{array}$ & $\begin{array}{c}41.84 \pm 5.01 \\
<40.0: 308(33.48 \%) \\
>50.0: 26(2.82 \%)\end{array}$ & $\begin{array}{c}40.34 \pm 3.35 \\
248(44.60 \%) \\
1(0.18 \%)\end{array}$ & $\begin{array}{c}44.14 \pm 6.13 \\
60(16.48 \%) \\
25(6.87 \%)\end{array}$ & $\begin{array}{c}P<0.001 \\
P\left(X^{2}\right)=0.0005 \\
P\left(X^{2}\right)=0.0005\end{array}$ \\
\hline $\begin{array}{l}\text { Hemoglobin }(\mathrm{Hb})(\mathrm{g} / \mathrm{dL}) \\
(13.5-17.0)\end{array}$ & $\begin{array}{c}13.98 \pm 1.39 \\
<13.5: 324(35.22 \%) \\
>17.0: 26(2.82 \%)\end{array}$ & $\begin{array}{c}13.50 \pm 1.14 \\
259(46.58 \%) \\
0(0 \%)\end{array}$ & $\begin{array}{c}14.72 \pm 1.42 \\
65(17.86 \%) \\
26(7.14 \%)\end{array}$ & $\begin{array}{c}P<0.001 \\
P\left(X^{2}\right)=0.0005 \\
P\left(X^{2}\right)=0.0005\end{array}$ \\
\hline $\begin{array}{l}\text { Mean Corpuscular Volume (MCV) (fL) } \\
\qquad(80-100)\end{array}$ & $\begin{array}{c}90.94 \pm 5.06 \\
<80: 16(1.74 \%) \\
>100: 32(3.48 \%)\end{array}$ & $\begin{array}{c}90.41 \pm 5.00 \\
9(1.62 \%) \\
10(1.80 \%)\end{array}$ & $\begin{array}{c}91.75 \pm 5.05 \\
7(1.92 \%) \\
22(6.04 \%)\end{array}$ & $\begin{array}{c}P<0.001 \\
P\left(X^{2}\right)=0.79 \\
P\left(X^{2}\right)=0.001\end{array}$ \\
\hline $\begin{array}{l}\text { Mean Corpuscular Hemoglobin }(\mathrm{MCH})(\mathrm{pg}) \\
\qquad(27.0-33.0)\end{array}$ & $\begin{array}{c}30.48 \pm 1.93 \\
<27.0: 31(3.37 \%) \\
>33.0: 67(7.28 \%)\end{array}$ & $\begin{array}{c}30.27 \pm 1.94 \\
21(3.77 \%) \\
44(7.91 \%)\end{array}$ & $\begin{array}{c}30.80 \pm 1.88 \\
10(2.74 \%) \\
23(6.32 \%)\end{array}$ & $\begin{array}{c}P<0.001 \\
P\left(X^{2}\right)=0.45 \\
P\left(X^{2}\right)=0.36\end{array}$ \\
\hline $\begin{array}{l}\text { Mean Corpuscular Hemoglobin Concentration } \\
(\mathrm{MCHC})(\mathrm{g} / \mathrm{dL}) \\
(31.0-35.0)\end{array}$ & $\begin{array}{c}33.46 \pm 1.25 \\
<31.0: 3(0.33 \%) \\
>35.0: 29(3.15 \%)\end{array}$ & $\begin{array}{c}33.40 \pm 1.50 \\
3(0.54 \%) \\
10(1.80 \%)\end{array}$ & $\begin{array}{c}33.56 \pm 0.71 \\
0(0 \%) \\
19(5.22 \%)\end{array}$ & $\begin{array}{c}P=0.13 \\
P\left(X^{2}\right)=0.28 \\
P\left(X^{2}\right)=0.004\end{array}$ \\
\hline $\begin{array}{l}\text { Red Blood Cell Distribution Width (RDW) (\%) } \\
\qquad(11.0-15.0)\end{array}$ & $\begin{array}{c}13.21 \pm 1.54 \\
<11.0: 5(0.54 \%) \\
>15.0: 61(6.63 \%)\end{array}$ & $\begin{array}{c}13.21 \pm 1.64 \\
4(0.72 \%) \\
40(7.19 \%)\end{array}$ & $\begin{array}{c}13.21 \pm 1.38 \\
1(0.27 \%) \\
21(5.77 \%)\end{array}$ & $\begin{array}{c}P=0.78 \\
P\left(X^{2}\right)=0.65 \\
P\left(X^{2}\right)=0.42\end{array}$ \\
\hline $\begin{array}{l}\text { White Blood Cell Count (WBC) }\left(\times 10^{3} / \mu \mathrm{L}\right) \\
\qquad(4.0-11.0)\end{array}$ & $\begin{array}{c}6.37 \pm 1.89 \\
<4.0: 49(5.33 \%) \\
>11.0: 16(1.74 \%)\end{array}$ & $\begin{array}{c}6.14 \pm 1.85 \\
36(6.47 \%) \\
8(1.44 \%)\end{array}$ & $\begin{array}{l}6.71 \pm 1.90 \\
13(3.57 \%) \\
8(2.20 \%)\end{array}$ & $\begin{array}{c}P<0.001 \\
P\left(X^{2}\right)=0.07 \\
P\left(X^{2}\right)=0.44\end{array}$ \\
\hline
\end{tabular}




\begin{tabular}{|c|c|c|c|c|}
\hline $\begin{array}{l}\% \text { Neutrophils } \\
(45.0-70.0)\end{array}$ & $\begin{array}{c}61.66 \pm 9.28 \\
<45.0: 33(3.58 \%) \\
>70.0: 173(18.80 \%)\end{array}$ & $\begin{array}{c}61.39 \pm 8.90 \\
19(3.42 \%) \\
100(17.98 \%)\end{array}$ & $\begin{array}{c}62.07 \pm 9.84 \\
14(3.84 \%) \\
73(20.05 \%)\end{array}$ & $\begin{array}{c}P=0.14 \\
P\left(X^{2}\right)=0.85 \\
P\left(X^{2}\right)=0.43\end{array}$ \\
\hline $\begin{array}{l}\text { \% Lymphocytes } \\
(20.0-40.0)\end{array}$ & $\begin{array}{c}28.80 \pm 8.20 \\
<20: 124(13.48 \%) \\
>40: 80(8.69 \%)\end{array}$ & $\begin{array}{l}29.52 \pm 8.02 \\
63(11.33 \%) \\
56(10.07 \%)\end{array}$ & $\begin{array}{c}27.69 \pm 8.37 \\
61(16.76 \%) \\
24(6.59 \%)\end{array}$ & $\begin{array}{c}P<0.001 \\
P\left(X^{2}\right)=0.02 \\
P\left(X^{2}\right)=0.07\end{array}$ \\
\hline $\begin{array}{l}\text { \% Monocytes } \\
(3.0-10.0)\end{array}$ & $\begin{array}{c}7.19 \pm 2.10 \\
<3.0: 11(1.19 \%) \\
>10.0: 79(8.58 \%)\end{array}$ & $\begin{array}{l}7.00 \pm 2.08 \\
11(1.98 \%) \\
38(6.83 \%)\end{array}$ & $\begin{array}{c}7.49 \pm 2.10 \\
0(0 \%) \\
41(11.26 \%)\end{array}$ & $\begin{array}{c}P<0.001 \\
P\left(X^{2}\right)=0.009 \\
P\left(X^{2}\right)=0.02\end{array}$ \\
\hline $\begin{array}{l}\text { \% Eosinophils } \\
(1.0-5.0)\end{array}$ & $\begin{array}{c}2.72 \pm 2.64 \\
<1.0: 48(5.22 \%) \\
>5.0: 60(6.52 \%)\end{array}$ & $\begin{array}{l}2.49 \pm 1.57 \\
33(5.93 \%) \\
24(4.32 \%)\end{array}$ & $\begin{array}{l}3.05 \pm 3.62 \\
15(4.12 \%) \\
36(9.89 \%)\end{array}$ & $\begin{array}{c}P=0.01 \\
P\left(X^{2}\right)=0.28 \\
P\left(X^{2}\right)=0.001\end{array}$ \\
\hline $\begin{array}{c}\text { \% Basophils } \\
(0.0-1.0)\end{array}$ & $\begin{array}{c}0.57 \pm 1.31 \\
>1.0: 33(3.58 \%)\end{array}$ & $\begin{array}{l}0.48 \pm 0.19 \\
10(1.80 \%)\end{array}$ & $\begin{array}{l}0.69 \pm 2.01 \\
23(6.32 \%)\end{array}$ & $\begin{array}{c}P<0.001 \\
P\left(X^{2}\right)=0.0005\end{array}$ \\
\hline $\begin{array}{l}\text { Platelet Count (PTL) }\left(\times 10^{3} / \mu \mathrm{L}\right) \\
(150-450)\end{array}$ & $\begin{array}{l}227.42 \pm 68.69 \\
<150: 75(8.15 \%) \\
>450: 6(0.65 \%)\end{array}$ & $\begin{array}{c}238.21 \pm 66.51 \\
30(5.39 \%) \\
4(0.72 \%)\end{array}$ & $\begin{array}{c}210.62 \pm 68.92 \\
45(12.36 \%) \\
2(0.55 \%)\end{array}$ & $\begin{array}{c}P<0.001 \\
P\left(X^{2}\right)=0.0005 \\
P\left(X^{2}\right)=0.91\end{array}$ \\
\hline $\begin{array}{l}\text { Mean Platelet Volume (MPV) (fL) } \\
\qquad(6.0-10.0)\end{array}$ & $\begin{array}{c}8.31 \pm 1.02 \\
<6.0: 4(0.43 \%) \\
>10.0: 58(6.30 \%)\end{array}$ & $\begin{array}{c}8.28 \pm 0.95 \\
1(0.18 \%) \\
29(5.21 \%)\end{array}$ & $\begin{array}{c}8.37 \pm 1.11 \\
3(0.82 \%) \\
29(7.97 \%)\end{array}$ & $\begin{array}{c}P=0.44 \\
P\left(X^{2}\right)=0.30 \\
P\left(X^{2}\right)=0.09\end{array}$ \\
\hline $\begin{array}{c}\text { BL-MMSE Score (30) } \\
<24 / 30 \\
>24 / 30\end{array}$ & $\begin{array}{c}19.63 \pm 7.17 \\
650(70.65 \%) \\
270(29.35 \%)\end{array}$ & $\begin{array}{c}18.78 \pm 7.11 \\
393(70.68 \%) \\
163(29.32 \%)\end{array}$ & $\begin{array}{l}20.92 \pm 7.09 \\
257(70.60 \%) \\
107(29.80 \%)\end{array}$ & $\begin{array}{c}P<0.001 \\
P\left(X^{2}\right)=0.97 \\
P\left(X^{2}\right)=0.97\end{array}$ \\
\hline BL-ADAS-Cognitive & $12.45 \pm 10.48$ & $12.71 \pm 10.37$ & $12.04 \pm 10.65$ & $P=0.11$ \\
\hline BL-ADAS-Memory & $13.59 \pm 4.99$ & $13.75 \pm 5.09$ & $13.35 \pm 4.84$ & $P=0.24$ \\
\hline BL-ADAS-Cognitive Total & $25.87 \pm 14.74$ & $26.30 \pm 14.58$ & $25.23 \pm 14.99$ & $P=0.14$ \\
\hline BL-ADAS-Non Cognitive & $5.93 \pm 4.74$ & $6.32 \pm 4.87$ & $5.32 \pm 4.47$ & $P<0.001$ \\
\hline BL-ADAS-Total & $31.77 \pm 17.58$ & $32.59 \pm 17.51$ & $30.53 \pm 17.67$ & $P=0.02$ \\
\hline
\end{tabular}

Data: mean $\pm S D$

Table 1: Sample features.

using a QIAamp DNA Micro Kit (QIAGEN, Hilden, Germany). To determine the APOE $\varepsilon 2, \varepsilon 3$ and $\varepsilon 4$ alleles, SNPs rs7412 and rs429358 were analyzed by allelic discrimination using the TaqMan realtime PCR system. Validated SNP genotyping assays (ID number C_3084793_20 for rs429358 and C_904973_10 for rs7412) were purchased from Applied Biosystems (Applied Biosystems, CA, USA). Both SNP genotyping reactions were optimized in a total volume of $15 \mu \mathrm{l}$ using $20 \mathrm{ng}$ of DNA, and fluorescence was detected in a StepOne Plus Real-Time PCR system (Applied Biosystems, CA, USA). Control samples with known APOE genotypes were included in each PCR run, which were analyzed by the alternative PCR-RFLP method.

\section{TOMM40 genotyping}

The chr19:50094819+50094997 (NCBI36/hg18 human genome assembly) fragment containing rs10524523 was amplified from genomic DNA samples (10-20 ng) with 5'- fluorescently labeled forward primer ACCTCAAGCTGTCCTCTTGC and unlabeled reverse primer GAGGCTGAGAAGGGAGGATT (each at $0.5 \mu \mathrm{M}$ concentration). The PCR reaction was performed with FailSafe PCR kit (Epicentre Biotechnologies, Madison, WI) using buffer G from the kit. The PCR reaction was carried out in $20 \mu$ l volumes under the following conditions: $3 \mathrm{~min}$ at $95^{\circ} \mathrm{C}, 40 \mathrm{~s}$ at $98^{\circ} \mathrm{C}$, then 10 cycles with $30 \mathrm{~s}$ at $95^{\circ} \mathrm{C}$, $90 \mathrm{~s}$ at $63^{\circ} \mathrm{C}$, then $18 \mathrm{cycles}$ with $30 \mathrm{~s}$ at $95^{\circ} \mathrm{C}, 1 \mathrm{~min}$ at $61^{\circ} \mathrm{C}$, then final extension $7 \mathrm{~min}$ at $72^{\circ} \mathrm{C}$. One microliter of each PCR product was diluted with $15 \mu \mathrm{l}$ of HiDi Formamide (Applied Biosystems, Foster city, CA) and supplemented with $0.3 \mu \mathrm{l}$ of $600 \mathrm{LIZ}$ size standard (Applied Biosystems, Foster city, CA). The size analysis was performed by Cornell University Genomics Core Laboratory (Ithaca, NY) on an ABI 3730 DNA analyzer (Applied Biosystems, Foster city, CA). ABI 3730 data were analyzed using GeneMarker software (SoftGenetics, State College, PA). The presence of poly T stretch in the fragment causes "stuttering" during the PCR reaction [74]. Therefore, an additional procedure was implemented in order to interpret complex peak patterns and to determine the genotypes with precision. Briefly, PCR products from several patients were subcloned and the length of the poly $\mathrm{T}$ stretch in each clone was determined by sequencing. A clone library that covered poly T lengths from 14 to 38 was generated. Plasmid DNA from each clone was diluted and PCR amplified under exactly the same conditions as described above. Peak patterns from PCR amplification of individual cloned DNA were compared to peak patterns obtained from each individual patient's DNA. The accuracy of the method was verified by determination of poly $\mathrm{T}$ lengths in coded samples of patients' DNA that were previously deep sequenced and coded by an independent group. Our method was found to be $100 \%$ accurate.

\section{Statistical analysis}

Statistical analysis (paired t-test, Analysis of Variance, $\chi^{2}$ and Fisher exact test, Mann-Withney Rank Sum test, Linear and Non-linear Regression analysis, Durbin-Watson statistic, Pearson correlation, Spearman rank) were performed by using the IBM SPSS Statistics 20 and Sigma Stat 3.5 programs, where appropriate. Results are expressed as mean \pm SD in the text and as mean \pm SEM in Figs. 2, 3 and 5 for clarity. A 2-tailed $\mathrm{p}<0.05$ (or $\mathrm{p}$ value $\left(\chi^{2}\right) \leq 0.05$ ) was considered statistically significant.

\section{Results}

\section{Structural features of the APOE-TOMM40 region}

The distribution and frequency of APOE genotypes was the following: APOE-2/3, 8.26\%; APOE-2/4, 1.96\%; APOE-3/3, 51.52\%; APOE-3/4, 33.04\%; and APOE-4/4, 5.22\%. The distribution of 6 major TOMM40 poly $\mathrm{T}$ variants was: $18.37 \% \mathrm{~S} / \mathrm{S}, 7.83 \% \mathrm{~S} / \mathrm{L}, 38.80 \% \mathrm{~S} / \mathrm{VL}$, $1.52 \% \mathrm{~L} / \mathrm{L}, 7.17 \% \mathrm{~L} / \mathrm{VL}$, and $26.31 \% \mathrm{VL} / \mathrm{VL}$ Figure 1 . The APOE-2/3 genotype was found to be associated with S/S (27.63\%), S/VL (51.32\%), and L/VL (21.05\%); APOE-2/4 was associated with S/L (16.67\%), S/ 
Citation: Cacabelos R, Goldgaber D, Vostrov A, Matsuki H, Torrellas C, et al. (2014) APOE-TOMM40 in the Pharmacogenomics of Dementia. J Pharmacogenomics Pharmacoproteomics 5: 135. doi:10.4172/2153-0645.1000135
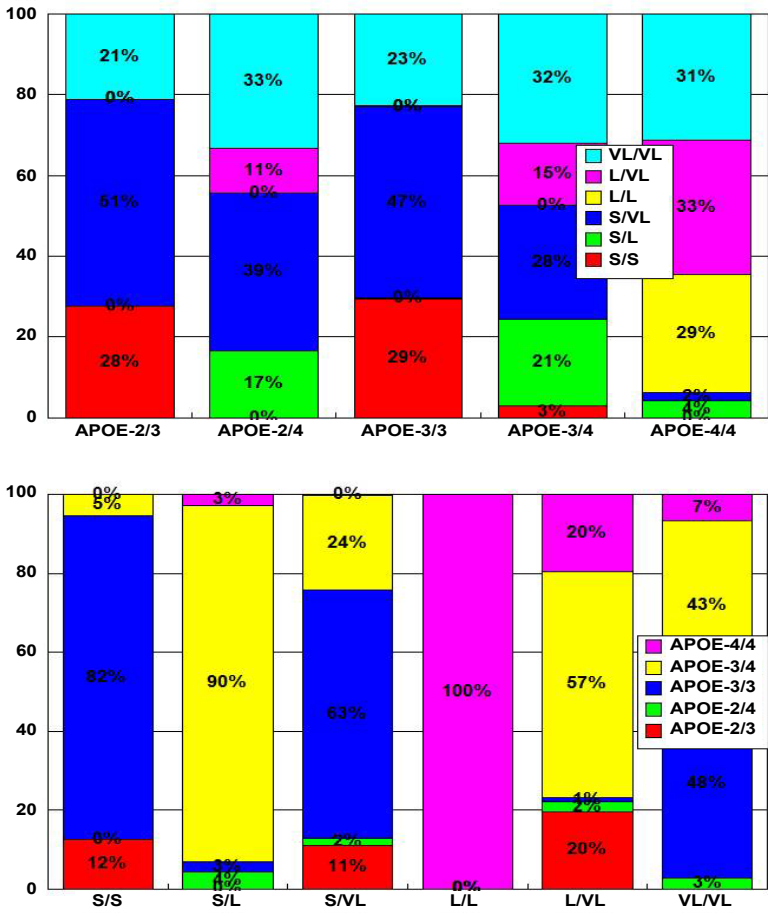

Figure 1: APOE-TOMM40 association.

Upper panel: Association of TOMM40 variants (S/S, S/L, S/VL, L/L, L/VL, VL/VL) with APOE-2/3, APOE-2/4, APOE-3/3, APOE-3/4 and APOE-4/4 genotypes.

VL (38.89\%), L/VL (11.11\%), and VL/VL (33.33\%); APOE-3/3 was associated with S/S (29.32\%), S/L (0.42\%), S/VL (47.26\%), L/VL (0.21\%), and VL/VL (22.79\%); APOE-3/4 was associated with S/S (2.96\%), S/L (21.38\%), S/VL (28.29\%), L/VL (15.46\%), and VL/VL (31.91\%); and APOE-4/4 was associated with S/L (4.17\%), S/VL (2.17\%), L/L (29.17\%), L/VL (33.33\%), and VL/VL (31.25\%). Likewise, the S/S genotype was associated with APOE-2/3 (27.63\%), 3/3 (29.32\%), and 3/4 (2.96\%); S/L with APOE-2/4 (16.67\%), 3/3 (0.42\%), 3/4 (21.38\%), and $4 / 4$ (4.17\%); S/VL with APOE-2/3 (51.32\%), $2 / 4$ (38.89\%), 3/3 (47.26\%), $3 / 4$ (28.29\%), and 4/4 (2.08\%); L/L was exclusively associated with APOE-4/4 (100\%); L/VL with APOE-2/4 (11.11\%), 3/3 (0.21\%), $3 / 4(15.46 \%)$, and $4 / 4$ (33.33\%); and VL/VL with APOE-2/3 (21.05\%), $2 / 4$ (33.33\%), 3/3 (22.79\%), 3/4 (31.91\%), and 4/4 (31.25\%) Figure 1. S/ $\mathrm{VL}$ and VL/VL are the only TOMM40 poly $\mathrm{T}$ genotypes which interact with all major APOE genotypes; in contrast, the APOE-4/4-TOMM40$\mathrm{L} / \mathrm{L}$ association is unique, representing approximately $30 \%$ of APOE$4 / 4$ carriers.

\section{Influence of APOE and TOMM40 poly $\mathrm{T}$ repeats on age at onset}

APOE-4/4 carriers were the youngest at age of onset $(69.95 \pm$ $7.10 \mathrm{yrs})$, showing a significant difference as compared with APOE$3 / 4$ carriers $(72.45 \pm 8.54$ yrs; $\mathrm{p}=0.008)$. APOE-3/3 carriers $(70.28 \pm$ 9.52 yrs, $\mathrm{p}<0.001)$ were also younger than APOE-3/4 carriers; and no differences were found between the age at onset of APOE-2/3 (71.43 \pm $8.64 \mathrm{yrs})$ or APOE- $2 / 4$ carriers $(71.77 \pm 11.05 \mathrm{yrs})$ and that of patients with other APOE genotypes. The age at onset of patients according to their TOMM40 poly T genotypes was as follows: S/S, $69.42 \pm 10.30 \mathrm{yrs}$; S/L, $72.37 \pm 7.46$ yrs; S/VL, $71.88 \pm 8.68$ yrs; L/L, $69.14 \pm 7.36$ yrs; L/VL,
$72.51 \pm 9.42$ yrs; and VL/VL, $70.51 \pm 9.07$ yrs. Significant differences were found between $\mathrm{S} / \mathrm{S}$ and $\mathrm{S} / \mathrm{VL}(\mathrm{p}=0.02), \mathrm{S} / \mathrm{VL}$ and VL/VL $(\mathrm{p}<0.05)$, $\mathrm{L} / \mathrm{L}$ and $\mathrm{L} / \mathrm{VL}(\mathrm{p}<0.05)$; and $\mathrm{L} / \mathrm{VL}$ vs $\mathrm{S} / \mathrm{S}(\mathrm{p}=0.03)$, and $\mathrm{L} / \mathrm{VL}$ vs VL/VL $(\mathrm{p}=0.04)$.

\section{Sex-related therapeutic response}

In the whole group Figure 2, there was a clear pattern in terms of cognitive performance, with a significant improvement in MMSE scores at $1(\mathrm{p}<0.001), 3(\mathrm{p}<0.001), 6(\mathrm{p}=0.008)$, and 9 months $(\mathrm{p}<0.05)$ of treatment. The peak effect was observed during the first 3 months of treatment with a gradual cognitive decline thereafter. This trend was almost identical in females and males Figure 2, with a response rate (\% of cases with a MMSE score superior or equal to baseline values after 12 months of treatment) of $60 \%$ in females and $59 \%$ in males $(59 \%$ responders (R) and $41 \%$ non-responders (NR) in the global sample), despite substantial gender-related differences in biochemical and hematological parameters Table 1; however, the degree of significance appeared to be more apparent in females than in males Figure 2.
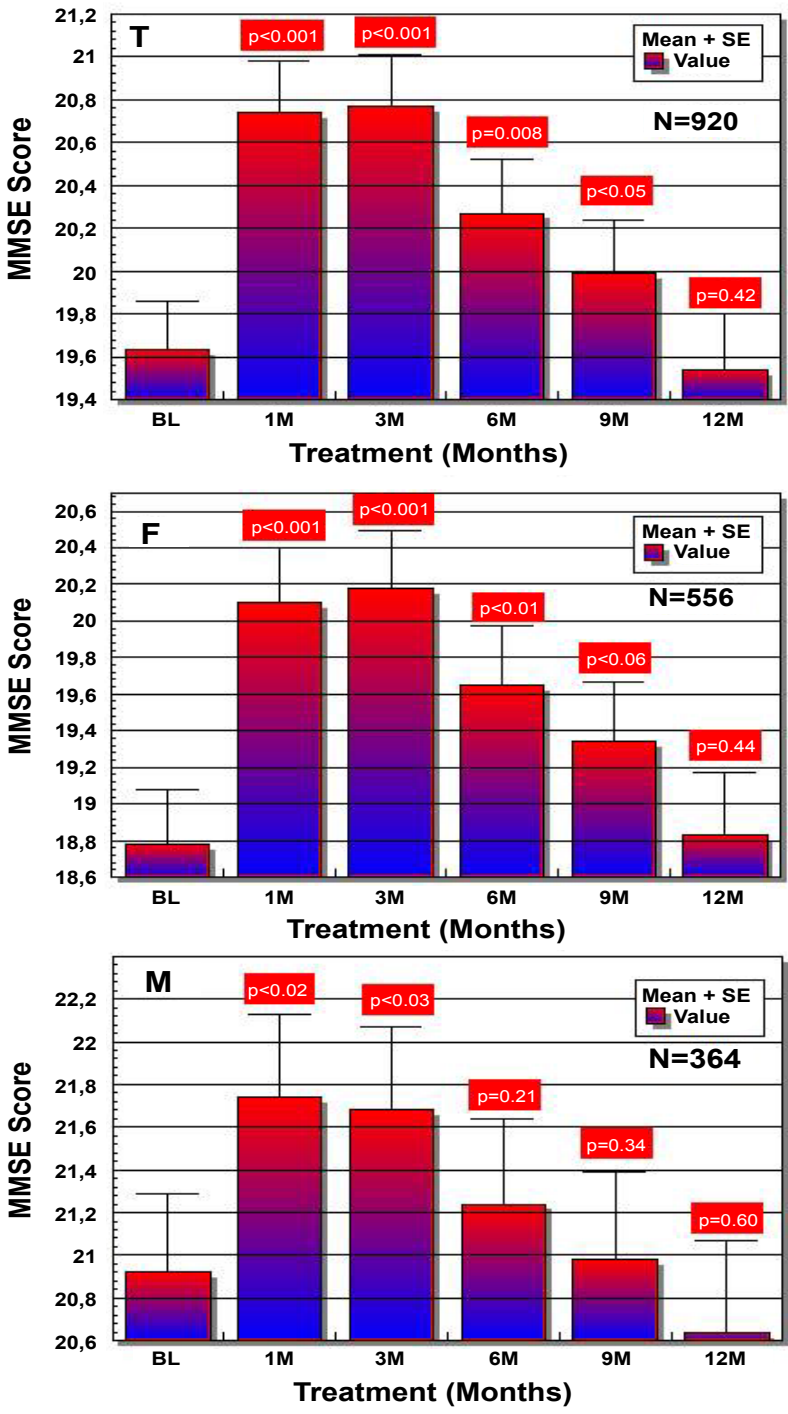

Figure 2: Therapeutic response to a multifactorial treatment in patients with Alzheimer's disease. ( $T$ ) Total $(N=920)$; $(F)$ Females $(N=556)$; (M) Males $(\mathrm{N}=364)$. BL: Baseline MMSE Score. Values: mean \pm se. 

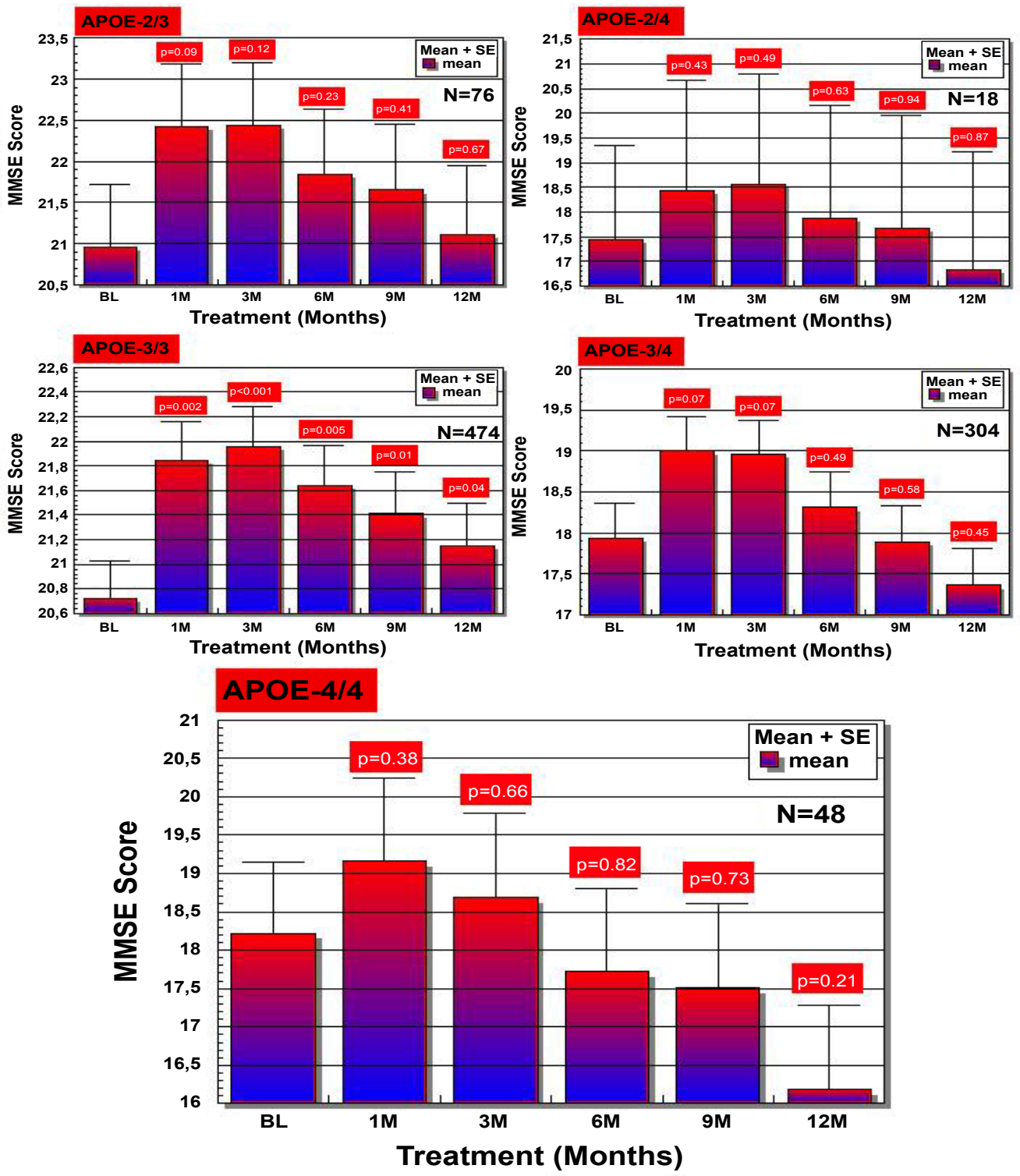

Figure 3: APOE-Related therapeutic response to a multifactorial treatment in patients with Alzheimer's disease.

BL: Baseline MMSE Score. Values: mean \pm se

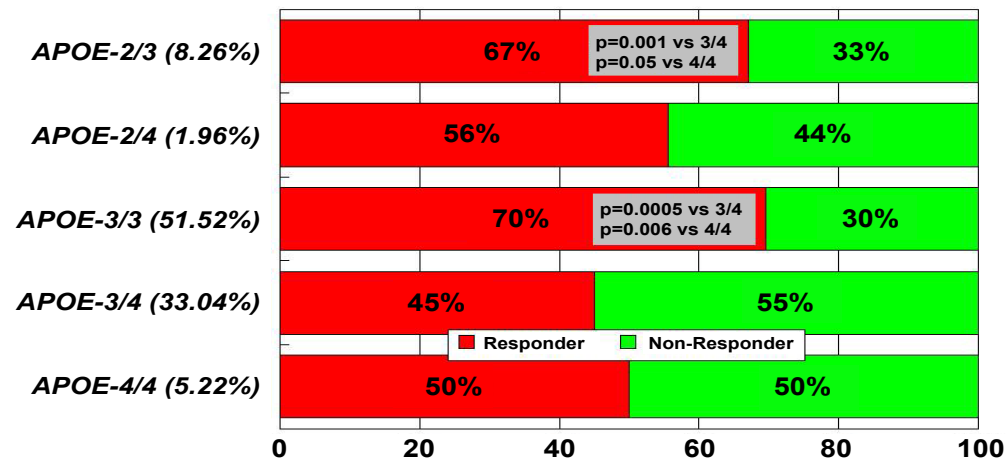

Figure 4: APOE-Related response rate (\%) in patients with Alzheimer's disease treated with a multifactorial therapy for one year. 
Citation: Cacabelos R, Goldgaber D, Vostrov A, Matsuki H, Torrellas C, et al. (2014) APOE-TOMM40 in the Pharmacogenomics of Dementia. J Pharmacogenomics Pharmacoproteomics 5: 135. doi:10.4172/2153-0645.1000135

\section{APOE- and TOMM40 poly T-related therapeutic response}

The same pattern of mental performance, with a transient profile of cognitive improvement for 6-12 months and maximum effect during the first 3 months of treatment, was observed in APOE-2/3, APOE-2/4, APOE-3/3, APOE-3/4, and APOE-4/4 carriers Figure 3, with significant effects in APOE-3/3 carriers for 12 months Figure 3. The response rate (RR) was $70 \%$ in APOE- $3 / 3,67 \%$ in APOE- $2 / 3,56 \%$ in APOE- $2 / 4,50 \%$ in APOE- $4 / 4$, and $45 \%$ in APOE-3/4 carriers Figure 4 , with significant differences between APOE- $2 / 3$ and APOE-3/4 ( $\mathrm{p}=0.001)$, APOE- $2 / 3$ and APOE-4/4 ( $\mathrm{p}=0.05)$, APOE-3/3 and APOE-3/4 $(\mathrm{p}>0.0005)$, and APOE- $3 / 3$ and APOE- $4 / 4\left(\mathrm{p}\left(\chi^{2}\right)<0.006\right)$ ( Figure 4.

The time-dependent profile of cognitive performance after treatment, according to the TOMM40 poly $\mathrm{T}$ genotype, was similar to that observed in the total group or in the APOE-related study, with an apparent improvement during the first 3-9 months of treatment; however, significant effects were only observed in patients harboring the TOMM40 poly T-S/S and S/VL genotypes Figure 5. S/S carriers were the best responders (70\%), followed by S/VL (61\%), VL/VL (57\%), and $\mathrm{L} / \mathrm{VL}$ carriers $(51 \%)$, and $\mathrm{L} / \mathrm{L}(35 \%)$ and S/L carriers $(45 \%)$ were the worst responders Figure 6 and 7.

\section{Discussion}

The present results appear to indicate that the combination therapy used in this study, probably together with the lipid-lowering effects and anti-atherosclerotic properties of sardilipin [18,24,73] and the prometabolic activity of animon complex, exert a positive synergistic effect on brain function accompanied by a transient improvement in mental performance, lasting for about 9-12 months Figure 2. The effect of CDP-choline, either alone or in combination with donepezil, as well as the effects of sardilipin, are APOE-dependent [3,18-28]. In our study, the administration of cholinesterase inhibitors was avoided in
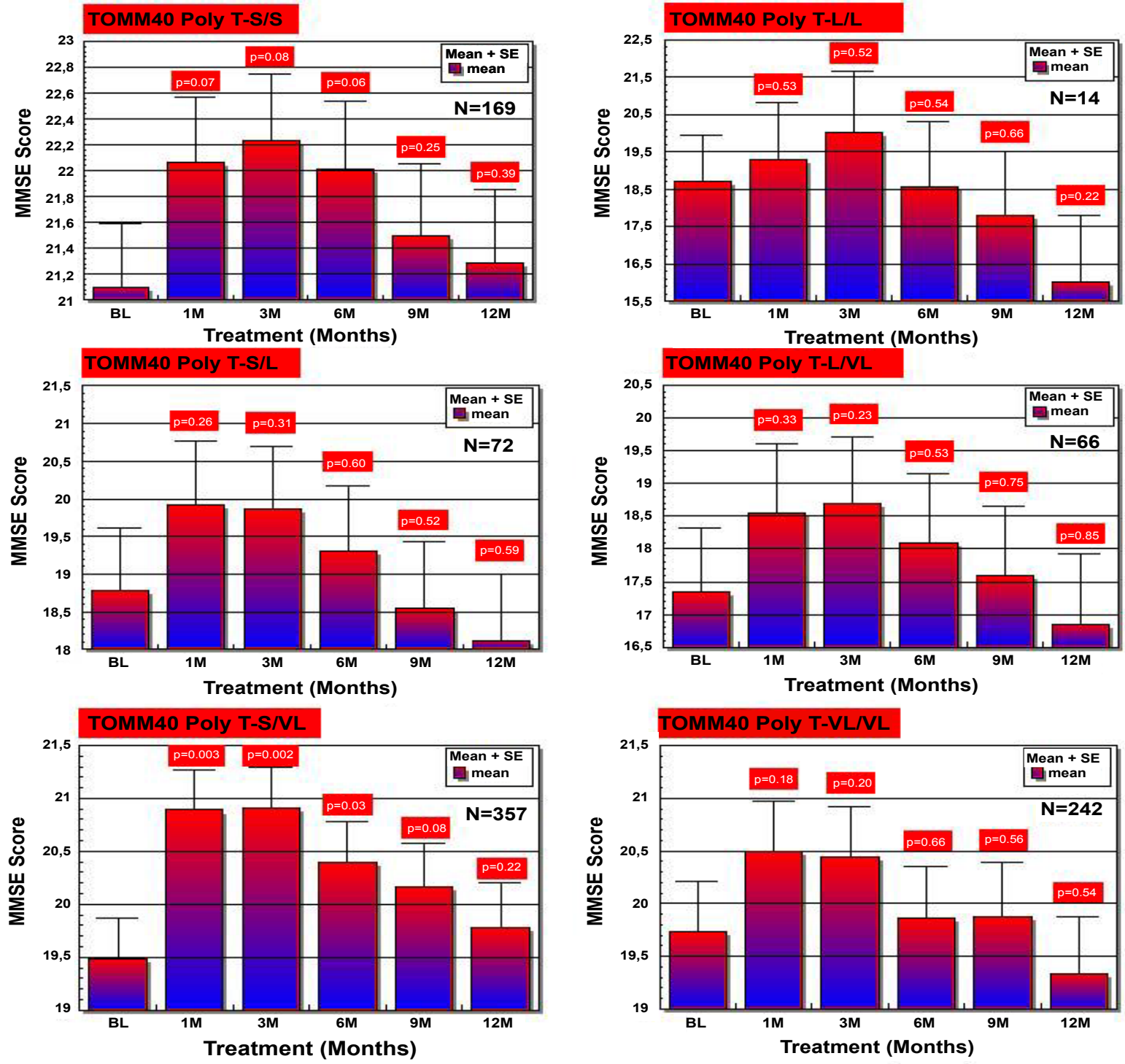

Figure 5: TOMM40-Related therapeutic response to a multifactorial treatment in patients with Alzheimer's disease. TOMM40 poly $T$ repeat variants: S/S, S/L, S/VL, L/L, L/VL, VL/VL.

BL: Baseline MMSE Score. Values: mean \pm se. 
Citation: Cacabelos R, Goldgaber D, Vostrov A, Matsuki H, Torrellas C, et al. (2014) APOE-TOMM40 in the Pharmacogenomics of Dementia. J Pharmacogenomics Pharmacoproteomics 5: 135. doi:10.4172/2153-0645.1000135

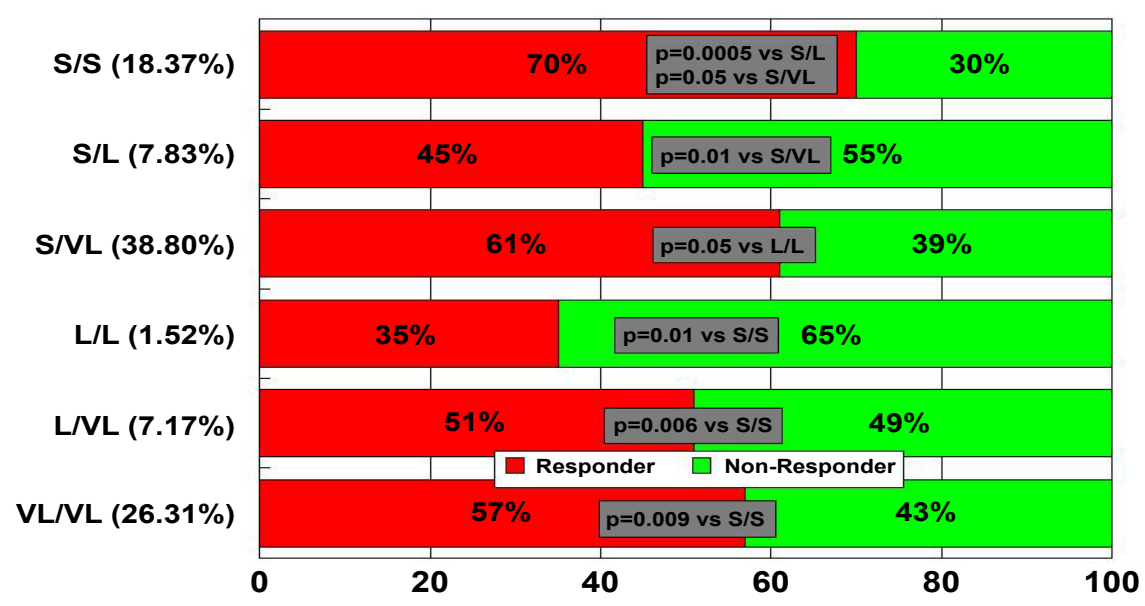

Figure 6: TOMM40-Related response rate (\%) in patients with Alzheimer's disease treated with a multifactorial therapy for one year.

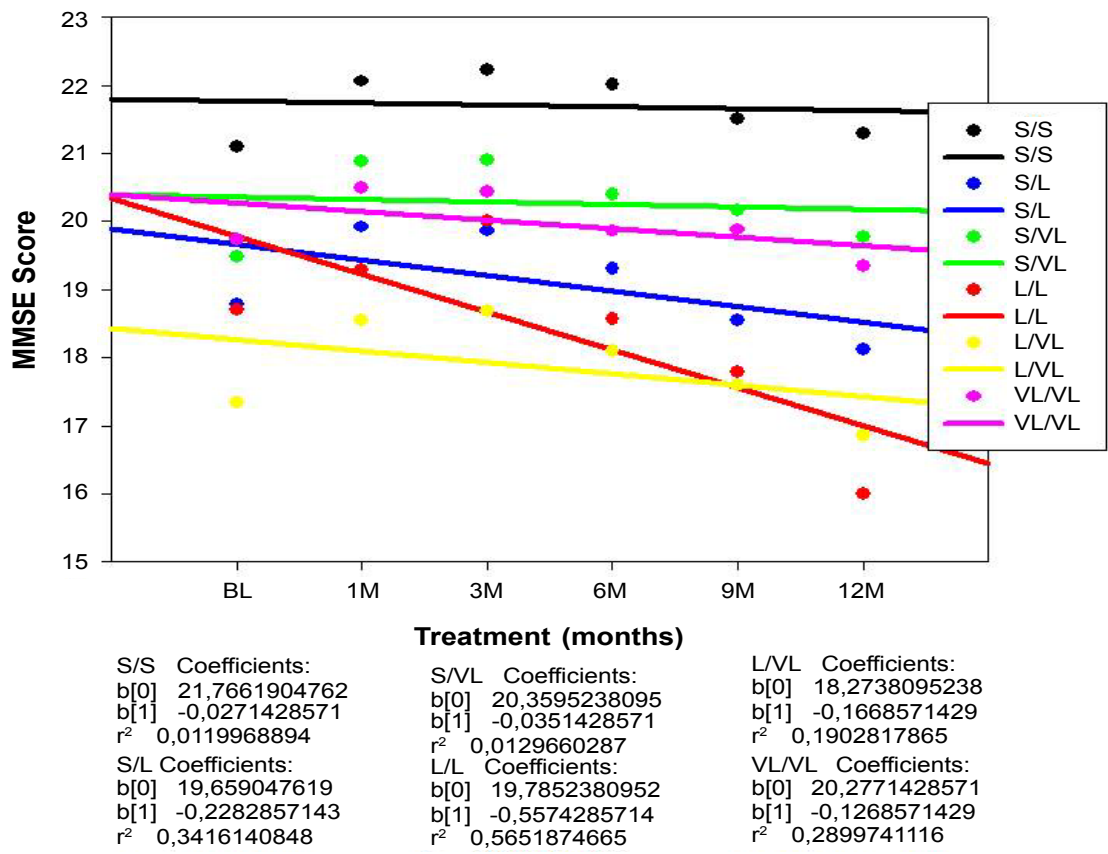

Figure 7: Comparative linear regression analysis of the cognitive response to a multifactorial treatment in patients with Alzheimer's disease according to their TOMM40 poly T variants.

BL: Baseline MMSE Score. Each point depicts the mean value of the MMSE scores in Figure 5 at baseline $(\mathrm{BL})$ and after $1,3,6,9$, and 12 months of treatment.

order to eliminate distorting variability and side-effects associated with CYP2D6-related factors $[25,27,75]$.

Our data also illustrate that polymorphic variants in the APOETOMM40 region are determinant for the therapeutic response to conventional drugs in patients with AD Figure 3-7. The role of APOE in this endeavor seems to be fundamental due to the influence of the APOE gene on the AD phenotype [76]; however, the impact of the TOMM40 gene on $\mathrm{AD}$ pathogenesis and pharmacogenetics is a novelty [33-36]. From studies designed to define $A P O E$-related AD phenotypes, several conclusions can be drawn: (i) the age-at-onset is 5-10 years earlier in approximately $80 \%$ of $\mathrm{AD}$ cases harboring the $A P O E-4 / 4$ genotype; (ii) the serum levels of ApoE are lowest in $A P O E-4 / 4$, intermediate in $A P O E-3 / 3$ and $A P O E-3 / 4$, and highest in $A P O E-2 / 3$ and $A P O E-2 / 4$; (iii) serum cholesterol levels are higher in $A P O E-4 / 4$ than in the other genotypes; (iv) HDL-cholesterol levels tend to be lower in $A P O E-3$ homozygotes than in APOE-4 allele carriers; (v) LDL-cholesterol levels are systematically higher in $A P O E-4 / 4$ than in any other genotype; (vi) triglyceride levels are significantly lower in $A P O E-4 / 4$; (vii) nitric oxide levels are slightly lower in $A P O E-4 / 4$; (viii) serum and CSF A $\beta$ levels tend to differ between $A P O E-4 / 4$ and the other most frequent genotypes (APOE-3/3, APOE-3/4); (ix) blood histamine levels are dramatically reduced in $A P O E-4 / 4$ as compared with the other genotypes; (x) 
brain atrophy and $\mathrm{AD}$ neuropathology is markedly increased in $A P O E-4 / 4>A P O E-3 / 4>A P O E-3 / 3$; (xi) brain mapping activity shows a significant increase in slow wave activity in $A P O E-4 / 4$ from early stages of the disease; (xii) brain hemodynamics, as reflected by reduced brain blood flow velocity and increased pulsatility and resistance indices, is significantly worse in APOE-4/4 (and in APOE-4 carriers in general, as compared with $A P O E-3$ carriers); brain hypoperfusion and neocortical oxygenation is also more deficient in APOE-4 carriers; (xiii) lymphocyte apoptosis is markedly enhanced in $A P O E-4$ carriers; (xiv) cognitive deterioration is faster in $A P O E-4 / 4$ patients than in carriers of any other APOE genotype; (xv) in approximately $3-8 \%$ of the $\mathrm{AD}$ cases, the presence of some dementia-related metabolic dysfunctions accumulates more in APOE-4 carriers than in APOE-3 carriers; (xvi) some behavioral disturbances, alterations in circadian rhythm patterns, and mood disorders are slightly more frequent in APOE-4 carriers; (xvii) aortic and systemic atherosclerosis is also more frequent in APOE-4 carriers; (xviii) liver metabolism and transaminase activity also differ in APOE-4/4 with respect to other genotypes; (xix) hypertension and other cardiovascular risk factors also accumulate in $A P O E-4$; and (xx) $A P O E-4 / 4$ carriers are the poorest responders to conventional drugs. These 20 major phenotypic features clearly illustrate the biological disadvantage of $A P O E-4$ homozygotes and the potential consequences that these patients may experience when they receive pharmacological treatment for $\mathrm{AD}$ and/or concomitant pathologies [1-5,18-30,77-79]. In our study, it is clear that APOE- 4 carriers are the worst responders to conventional treatments Figures 3 and 4.

Linnertz et al. [39] defined 3 allele groups for rs10524523 ('523'), based on the number of ' $T$ '-residues: 'Short' $(\mathrm{S}, \mathrm{T} \leq 19)$, 'Long' (L, 20 $\leq \mathrm{T} \leq 29$ ) and 'Very Long' (VL, $\mathrm{T} \geq 30$ ). Roses et al. [33-36] reported that longer lengths of rs 10524523 are associated with a higher risk for LOAD; for APOE-3/4 patients who developed LOAD after 60 years of age, individuals with long poly $\mathrm{T}$ repeats (19-39 nucleotides) linked to APOE-3 develop LOAD on an average of 7 years earlier than individuals with shorter poly $\mathrm{T}$ repeats (11-16 nucleotides) linked to APOE-3 $[33,34,37]$. Apparently, these results could not be replicated by other authors [46-49]. In our case, we clearly found that patients harboring the APOE-4/4-L/L cluster developed dementia at an earlier age $(<70$ yrs) than their counterparts with other genotypes. In fact, $L / L$ carriers were the youngest at age of onset, followed by S/S carriers. In addition, virtually $100 \%$ of $\mathrm{L} / \mathrm{L}$ carriers were exclusively associated with APOE4/4 Figure 1, representing the worst responders to our combination therapy Figure 6. The APOE-3/3-VL/VL cluster, with an earlier age at onset (mean age $\sim 70 \mathrm{yrs}$ ), was present in approximately a quarter of APOE-3/3 carriers Figure 1.

Bernardi et al. [45] studied the association between TOMM40 rs10524523, age of onset, and memory performance in patients with the PSEN1 M146L mutation in a large familial AD Calabrian kindred, and found that APOE33/TOMM40VL/VL patients showed a tendency for an earlier age at onset compared to those with APOE33/TOMM40VL/S and APOE33/TOMM40S/S. TOMM40VL/VL patients had better memory performance, when compared to TOMM40S/S but not to TOMM40VL/S patients. For Li et al. [46], TOMM40 intron 6 poly $\mathrm{T}$ length may explain some of the variation in age at onset in PSEN2 familial $\mathrm{AD}$ and may be associated with $\mathrm{AD}$ neuropathology in persons with APOE-3/3.

The allele distribution of TOMM40 poly T repeats in the Spanish population reflects a high proportion of heterozygous S/VL (39\%), followed by homozygous VL (27\%) and homozygous S (19\%). Homozygous L/L represents $1.52 \%$ of the Spanish population, and both S/VL and L/VL genotypes conform a group of about 7-8\% of the population Figure 6. Potential dissimilarities with other White and Hispanic populations [39], might be due to the ancestral admixture of different cultures in the Iberian peninsula. The linkage pattern between TOMM40-'523' and APOE alleles in Whites and Hispanics reflects that the $\mathrm{L}$ is primarily linked to APOE-4, while the majority of the $\mathrm{VL}$ and $\mathrm{S}$ are linked to APOE-3. In African-Americans, Ghanaians and Japanese, there is an increased frequency of the '523'S-APOE-4 haplotype [39].

Several reports suggest that both APOE and TOMM40 influence memory performance in normal [53] and pathological conditions $[54,80]$. For some authors, both TOMM40 and APOE significantly influence age-related memory performance, but they appear to do so independently of each other [80]. Others suggest important APOEindependent associations between the TOMM40 '523' polymorphism and specific cognitive domains of memory and executive control that are preferentially affected in early-stage $\mathrm{AD}$, with $\mathrm{S}$ homozygotes performing better than the S/L-S/VL and the VL/L-L/VL-VL/VL genotype groups on measures associated with memory and executive function [54]. According to our data, the best mental performance (and response rate to treatment) is observed in patients harboring the APOE3/3-S/S haplotype (R 70\%), followed by those with the APOE-3/3-S/ VL haplotype (R 60\%)(Figs. 3-6). In general, S/S carriers are the best responders $>$ S/VL $(61 \%)>$ VL/VL $(57 \%)>$ L/VL $(51 \%)>$ S/L $(45 \%)>$ L/L (35\%) Figure 6. The presence of the L allele appears to contribute to a poor therapeutic outcome, and when the L/L genotype associates with the APOE- $4 / 4$ genotype, carriers of the APOE-4/4-S/S haplotype ( $30 \%$ of APOE- $4 / 4$ carriers) are converted into the worst responders.

Although a retrospective study such as this, designed to assess the potential influence of the APOE-TOMM40 region on cognitive performance in response to a heterogeneous multifactorial therapy in a natural setting, has its limitations, interpretation of the obtained results appears to indicate that the APOE-TOMM40 cluster is important in both the clinical course of the disease and the biological conditions of $\mathrm{AD}$ patients in their response to the different drugs necessary to protect brain function, transiently halting disease progression. The relevance of the APOE-TOMM40 locus in the complex phenotype of dementia might be due to the pleiotropic properties of these genes. Although a large number of pharmacogenetic studies in AD used APOE as a reference gene with plural results $[3,76]$, more specific studies exploring the influence of APOE, TOMM40, CYP, and ABCB1 variants on the pharmacogenetic outcome are needed to optimize therapeutics in $\mathrm{AD}$ $[3,35,81]$.

\section{Conclusions}

1. A multifactorial treatment with conventional neuroprotectants and other drugs or metabolic factors to treat concomitant pathologies o metabolic deficiencies, respectively, seems to be useful for patients with dementia in approximately $50 \%$ of the cases, stabilizing or improving cognitive deterioration for a transient period of time ( $<12$ months).

2. APOE- 4 carriers are the worst responders and APOE- 3 carriers are the best responders to conventional treatments.

3. TOMM40 poly T-S/S carriers are the best responders, VL/VL and S/VL carriers are intermediate responders, and L/L carriers are the worst responders to treatment.

4. Patients harboring a large (L) number of poly $\mathrm{T}$ repeats in intron 6 of the TOMM40 gene (L/L or S/L genotypes) in haplotypes associated with APOE-4 are the worst responders to treatment. 
Citation: Cacabelos R, Goldgaber D, Vostrov A, Matsuki H, Torrellas C, et al. (2014) APOE-TOMM40 in the Pharmacogenomics of Dementia. J Pharmacogenomics Pharmacoproteomics 5: 135. doi:10.4172/2153-0645.1000135

5. Patients with short (S) TOMM40 poly T variants (S/S genotype), and to a lesser extent S/VL and VL/VL carriers, in haplotypes with APOE-3 are the best responders to treatment.

6. In $100 \%$ of the cases, the $\mathrm{L} / \mathrm{L}$ genotype is exclusively associated with the APOE-4/4 genotype, and this haplotype (4/4-L/L) is probably responsible for early onset of the disease, a faster cognitive decline, and a poor response to different treatments.

\section{References}

1. Cacabelos R (2011) Alzheimer's disease 2011: where are we heading?. Gen-T 8: $54-86$

2. Cacabelos R (2012) Pharmacogenomics of central nervous system (CNS) drugs. Drug Dev Res 73: 461-476

3. Cacabelos R, Cacabelos P, Torrellas C, Tellado I, Carril JC (In press) Pharmacogenomics of Alzheimer's disease. Novel therapeutic strategies for Drug development. Methods Mol Biol.

4. Cacabelos R, López-Muñoz F (2014) The ABCB1 transporter in Alzheimer's disease. Clin Exp Pharmacol 4: e128.

5. Cacabelos R, Fernandez-Novoa L, Lombardi V, Kubota Y, Takeda M (2005) Molecular genetics of Alzheimer's disease and aging. Methods Find Exp Clin Pharmacol 27 Suppl A: 1-573.

6. Bertram L, McQueen MB, Mullin K, Blacker D, Tanzi RE (2007) Systematic meta-analyses of Alzheimer disease genetic association studies: the AlzGene database. Nat Genet 39: 17-23

7. Selkoe DJ, Podlisny MB (2002) Deciphering the genetic basis of Alzheimer's disease. Annu Rev Genomics Hum Genet 3: 67-99.

8. Suh YH, Checler F (2002) Amyloid precursor protein, presenilins, and alphasynuclein: molecular pathogenesis and pharmacological applications in Alzheimer's disease. Pharmacol Rev 54: 469-525.

9. Larner AJ (2013) Presenilin-1 mutations in Alzheimer's disease: an update on genotype-phenotype relationships. J Alzheimers Dis 37: 653-659.

10. Hardy J, Selkoe DJ (2002) The amyloid hypothesis of Alzheimer's disease: progress and problems on the road to therapeutics. Science 297: 353-356.

11. www.alzgene.org

12. Hooli BV, Kovacs-Vajna ZM2, Mullin K, Blumenthal MA, Mattheisen M3, et al. (2014) Rare autosomal copy number variations in early-onset familial Alzheimer's disease. Mol Psychiatry 19: 676-681.

13. Roses AD, Strittmatter WJ, Pericak-Vance MA, Corder EH, Saunders AM, et al. (1994) Clinical application of apolipoprotein E genotyping to Alzheimer's disease. Lancet 343: 1564-1565.

14. Schupf N, Barral S, Perls T, Newman A, Christensen K, et al. (2013) Apolipoprotein E and familial longevity. Neurobiol Aging 34: 1287-1291.

15. Li YJ, Scott WK, Hedges DJ, Zhang F, Gaskell PC, et al. (2002) Age at onset in two common neurodegenerative diseases is genetically controlled. Am J Hum Genet 70: 985-993

16. Corder EH, Saunders AM, Risch NJ, Strittmatter WJ, Schmechel DE, et al (1994) Protective effect of apolipoprotein E type 2 allele for late onset Alzheimer disease. Nat Genet 7: 180-184.

17. Corder EH, Saunders AM, Strittmatter WJ, Schmechel DE, Gaskell PC, et al (1993) Gene dose of apolipoprotein E type 4 allele and the risk of Alzheimer's disease in late onset families. Science 261: 921-923.

18. Cacabelos R, Takeda M (2006) Pharmacogenomics, nutrigenomics and future therapeutics in Alzheimer's disease. Drugs Future 31(Suppl B): 5-146.

19. Cacabelos R (2003) The application of functional genomics to Alzheimer's disease. Pharmacogenomics 4: 597-621.

20. Cacabelos R (2008) Pharmacogenomics in Alzheimer's disease. Methods Mol Biol 448: 213-357.

21. Cacabelos R, Martínez-Bouza R (2011) Genomics and pharmacogenomics of dementia. CNS Neurosci Ther 17: 566-576.

22. Cacabelos $R$ (2011) The path to personalized medicine in mental disorders In: Ritsner MS (Ed) The handbook of neuropsychiatric biomarkers, endophenotypes and genes. Springer 4: 3-63.
23. Cacabelos $R$ (2009) Pharmacogenomics and therapeutic strategies for dementia. Expert Rev Mol Diagn 9: 567-611.

24. Cacabelos R, Fernández-Novoa L, Martínez-Bouza R, McKay A, Carril JC et al. (2010) Future trends in the pharmacogenomics of brain disorders and dementia: Influence of APOE and CYP2D6 variants. Pharmaceuticals 3: 3040 3100 .

25. Cacabelos R (2007) Donepezil in Alzheimer's disease: From conventional trials to pharmacogenetics. Neuropsychiatr Dis Treat 3: 303-333.

26. Cacabelos R, Llovo R, Fraile C, Fernández-Novoa L (2007) Pharmacogenetic aspects of therapy with cholinesterase inhibitors: the role of CYP2D6 in Alzheimer's disease pharmacogenetics. Curr Alzheimer Res 4: 479-500.

27. Cacabelos R (2007) Molecular pathology and pharmacogenomics in Alzheimer's disease: polygenic-related effects of multifactorial treatments on cognition, anxiety and depression. Methods Find Exp Clin Pharmacol 29 Suppl A: 1-91.

28. Cacabelos R, Fernández-Novoa L, Pichel V, Lombardi V, Kubota Y, et al. (2004) Pharmacogenomic studies with a combination therapy in Alzheimer's disease. Molecular Neurobiology of Alzheimer Disease and Related Disorders. Karger: Basel, Switzerland, pp. 94-107.

29. Roses AD (2004) Pharmacogenetics and drug development: the path to safer and more effective drugs. Nat Rev Genet 5: 645-656.

30. Roses AD (2008) Pharmacogenetics in drug discovery and development: a translational perspective. Nat Rev Drug Discov 7: 807-817.

31. Takei N, Miyashita A, Tsukie T, Arai H, Asada T, et al. (2009) Genetic association study on in and around the APOE in late-onset Alzheimer disease in Japanese. Genomics 93: 441-448.

32. Potkin SG, Guffanti G, Lakatos A, Turner JA, Kruggel F, et al. (2009) Hippocampal atrophy as a quantitative trait in a genome-wide association study identifying novel susceptibility genes for Alzheimer's disease. PLoS One 4: e6501.

33. Roses AD (2010) An inherited variable poly-T repeat genotype in TOMM40 in Alzheimer disease. Arch Neurol 67: 536-541.

34. Roses AD, Lutz MW, Amrine-Madsen H, Saunders AM, Crenshaw DG, et al (2010) A TOMM40 variable-length polymorphism predicts the age of late-onse Alzheimer's disease. Pharmacogenomics J 10: 375-384.

35. Roses AD, Saunders AM2, Lutz MW2, Zhang N3, Hariri AR2, et al. (2014) New applications of disease genetics and pharmacogenetics to drug development Curr Opin Pharmacol 14: 81-89.

36. Roses AD, Lutz MW, Crenshaw DG, Grossman I, Saunders AM, et al. (2013) TOMM40 and APOE: Requirements for replication studies of association with age of disease onset and enrichment of a clinical trial. Alzheimers Dement 9 : 132-136.

37. Lutz MW, Crenshaw DG, Saunders AM, Roses AD (2010) Genetic variation at a single locus and age of onset for Alzheimer's disease. Alzheimers Dement 6: $125-131$.

38. Shen L, Kim S, Risacher SL, Nho K, Swaminathan S, et al. (2010) Whole genome association study of brain-wide imaging phenotypes for identifying quantitative trait loci in $\mathrm{MCl}$ and $\mathrm{AD}$ : A study of the ADNI cohort. Neuroimage 53: 1051-1063.

39. Linnertz C, Saunders AM, Lutz MW, Crenshaw DM, Grossman I, et al (2012) Characterization of the poly-T variant in the TOMM40 gene in diverse populations. PLoS One 7: e30994.

40. Omoumi A, Fok A, Greenwood T2, Sadovnick AD2, Feldman HH, et al. (2014) Evaluation of late-onset Alzheimer disease genetic susceptibility risks in a Canadian population. Neurobiol Aging 35: 936.

41. Bagnoli S, Piaceri I, Tedde A Bessi V, Bracco L, et al. (2013) TOMM40 polymorphisms in Italian Alzheimer's disease and frontotemporal dementia patients. Neurol Sci 34: 995-998.

42. Ma XY, Yu JT, Wang W, Wang HF, Liu QY, et al. (2013) Association of TOMM40 polymorphisms with late-onset Alzheimer's disease in a Northern Han Chinese population. Neuromolecular Med 15: 279-287.

43. Valant V, Keenan BT, Anderson CD, Shulman JM, Devan WJ, et al. (2012) TOMM40 in Cerebral Amyloid Angiopathy Related Intracerebral Hemorrhage: Comparative Genetic Analysis with Alzheimer's Disease. Transl Stroke Res 3: 102-112. 
Citation: Cacabelos R, Goldgaber D, Vostrov A, Matsuki H, Torrellas C, et al. (2014) APOE-TOMM40 in the Pharmacogenomics of Dementia. J Pharmacogenomics Pharmacoproteomics 5: 135. doi:10.4172/2153-0645.1000135

44. Vounou M, Janousova E, Wolz R, Stein JL, Thompson PM, et al. (2012) Sparse reduced-rank regression detects genetic associations with voxel-wise longitudinal phenotypes in Alzheimer's disease. Neuroimage 60: 700-716.

45. Bernardi L, Gallo M, Anfossi M, Conidi ME, Colao R, et al. (2013) Role of TOMM40 rs10524523 polymorphism in onset of alzheimer's disease caused by the PSEN1 M146L mutation. J Alzheimers Dis 37: 285-289

46. Li G, Bekris LM, Leong L, Steinbart EJ, Shofer JB, et al. (2013) TOMM40 intron 6 poly-T length, age at onset, and neuropathology of $A D$ in individuals with APOE În3/În3. Alzheimers Dement 9: 554-561.

47. Jun G, Vardarajan BN, Buros J, Yu CE, Hawk MV, et al. (2012) Comprehensive search for Alzheimer disease susceptibility loci in the APOE region. Arch Neurol 69: $1270-1279$.

48. Cruchaga C, Nowotny P, Kauwe JS, Ridge PG, Mayo K, et al. (2011) Association and expression analyses with single-nucleotide polymorphisms in TOMM40 in Alzheimer disease. Arch Neurol 68: 1013-1019.

49. Chu SH, Roeder K, Ferrell RE, Devlin B, DeMichele-Sweet MA, et al. (2011) TOMM40 poly-T repeat lengths, age of onset and psychosis risk in Alzheimer disease. Neurobiol Aging 32: 2328.

50. Sebastiani P, Solovieff N, Dewan AT, Walsh KM, Puca A, et al. (2012) Genetic signatures of exceptional longevity in humans. PLoS One 7: e29848.

51. Beekman M, Blanché H, Perola M, Hervonen A, Bezrukov V, et al. (2013) Genome-wide linkage analysis for human longevity: Genetics of Healthy Aging Study. Aging Cell 12: 184-193.

52. Zhang C, Pierce BL (2014) Genetic susceptibility to accelerated cognitive decline in the US Health and Retirement Study. Neurobiol Aging 35: 1512.

53. Davies G, Harris SE, Reynolds CA, Payton A, Knight HM, et al. (2014) A genome-wide association study implicates the APOE locus in nonpathological cognitive ageing. Mol Psychiatry 19: 76-87.

54. Hayden KM, McEvoy JM, Linnertz C, Attix D, Kuchibhatla M, et al. (2012) A homopolymer polymorphism in the TOMM40 gene contributes to cognitive performance in aging. Alzheimers Dement 8: 381-388.

55. Linnertz C, Anderson L, Gottschalk W, Crenshaw D, Lutz MW, et al. (2014) The cis-regulatory effect of an Alzheimer's disease-associated poly-T locus on expression of TOMM40 and apolipoprotein E genes. Alzheimers Dement .

56. Lyall DM, Harris SE, Bastin ME, Muñoz Maniega S, Murray C, et al. (2014) Alzheimer's disease susceptibility genes APOE and TOMM40, and brain white matter integrity in the Lothian Birth Cohort 1936. Neurobiol Aging 35: 1513.

57. Johnson SC, La Rue A, Hermann BP, Xu G, Koscik RL, et al. (2011) The effect of TOMM40 poly-T length on gray matter volume and cognition in middle-aged persons with APOE Î $\mu 3 / \hat{\mu} \mu 3$ genotype. Alzheimers Dement 7: 456-465

58. Elias-Sonnenschein LS, Helisalmi S, Natunen T, Hall A, Paajanen T, et al. (2013) Genetic loci associated with Alzheimer's disease and cerebrospinal fluid biomarkers in a Finnish case-control cohort. PLoS One 8: e59676.

59. Silver M, Janousova E, Hua X, Thompson PM, Montana G; Alzheimer's Disease Neuroimaging Initiative (2012) Identification of gene pathways implicated in Alzheimer's disease using longitudinal imaging phenotypes with sparse regression. Neuroimage 63: 1681-1694.

60. Kim S, Swaminathan S, Shen L, Risacher SL, Nho K, et al. (2011) Genomewide association study of CSF biomarkers Abeta1-42, t-tau, and p-tau181p in the ADNI cohort. Neurology 76: 69-79.

61. Bruno D, Pomara N, Nierenberg J, Ritchie JC, Lutz MW, et al. (2012) Levels of cerebrospinal fluid neurofilament light protein in healthy elderly vary as a function of TOMM40 variants. Exp Gerontol 47: 347-352.

62. McFarquhar M, Elliott R, McKie S, Thomas E, Downey D, et al. (2014) TOMM40 rs2075650 may represent a new candidate gene for vulnerability to major depressive disorder. Neuropsychopharmacology 39: 1743-1753.

63. Mastaglia FL, Rojana-udomsart A, James I, Needham M, Day TJ, et al. (2013) Polymorphism in the TOMM40 gene modifies the risk of developing sporadic inclusion body myositis and the age of onset of symptoms. Neuromuscul Disord 23: 969-974

64. Seripa D, Bizzarro A, Pilotto A, Palmieri O, Panza F, et al. (2012) TOMM40, $\mathrm{APOE}$, and APOC1 in primary progressive aphasia and frontotemporal dementia. J Alzheimers Dis 31: 731-740.
65. Ellis J, Lange EM, Li J, Dupuis J, Baumert J, et al. (2014) Large multiethnic Candidate Gene Study for C-reactive protein levels: identification of a nove association at CD36 in African Americans. Hum Genet.

66. Zhou L, He M, Mo Z, Wu C, Yang H, et al. (2013) A genome wide association study identifies common variants associated with lipid levels in the Chinese population. PLoS One 8: e82420.

67. Zhang Z, Tao L, Chen Z, Zhou D, Kan M, et al. (2011) Association of genetic loc with blood lipids in the Chinese population. PLoS One 6: e27305.

68. Jiang R, Brummett BH, Hauser ER, Babyak MA, Siegler IC, et al. (2013) Chronic family stress moderates the association between a TOMM40 variant and triglyceride levels in two independent Caucasian samples. Biol Psychol 93: $184-189$

69. Guo Y, Lanktree MB, Taylor KC, Hakonarson H, Lange LA, et al. (2013) Genecentric meta-analyses of 108912 individuals confirm known body mass index loci and reveal three novel signals. Hum Mol Genet 22: 184-201.

70. Middelberg RP, Ferreira MA, Henders AK, Heath AC, Madden PA, et al. (2011) Genetic variants in LPL, OASL and TOMM40/APOE-C1-C2-C4 genes are associated with multiple cardiovascular-related traits. BMC Med Genet 12: 123.

71. Jeemon P, Pettigrew K, Sainsbury C, Prabhakaran D, Padmanabhan S (2011) Implications of discoveries from genome-wide association studies in current cardiovascular practice. World J Cardiol 3: 230-247.

72. Ronald J, Rajagopalan R, Ranchalis JE, Marshall JK, Hatsukami TS, et al. (2009) Analysis of recently identified dyslipidemia alleles reveals two loci that contribute to risk for carotid artery disease. Lipids Health Dis 8: 52.

73. Cacabelos R (2012) Sardilipin. In: Cacabelos R (Ed) World Guide for Drug Use and Pharmacogenomics. EuroEspes Publishing, Corunna, pp. 675.

74. Fazekas A, Steeves R, Newmaster S (2010) Improving sequencing quality from $\mathrm{PCR}$ products containing long mononucleotide repeats. Biotechniques 48: 277 285.

75. Noetzli M, Guidi M, Ebbing K, Eyer S, Wilhelm L, et al. (2014) Population pharmacokinetic approach to evaluate the effect of CYP2D6, CYP3A, ABCB POR and NR1/2 genotypes on donepezil clearance. Br J Clin Pharmacol 78 135-144.

76. Cacabelos R, Fernández-Novoa L, Carril JC, Carrera I, Corzo L, et al. (2012) APOE. In: Cacabelos R (Ed) World Guide for Drug Use and Pharmacogenomics. EuroEspes Publishing, Corunna, pp. 1121-1161.

77. Sabbagh MN, Malek-Ahmadi M, Dugger BN, Lee K, Sue LI, et al. (2013) The influence of Apolipoprotein E genotype on regional pathology in Alzheimer's disease. BMC Neurol 13: 44

78. Kennedy RE, Cutter GR, Schneider LS (2014) Effect of APOE genotype status on targeted clinical trials outcomes and efficiency in dementia and mild cognitive impairment resulting from Alzheimer's disease. Alzheimers Dement 10: $349-359$

79. Schiepers OJ, Harris SE, Gow AJ, Pattie A, Brett CE, et al. (2012) APOE E4 status predicts age-related cognitive decline in the ninth decade: longitudinal follow-up of the Lothian Birth Cohort 1921. Mol Psychiatry 17: 315-324.

80. Caselli RJ, Dueck AC, Huentelman MJ, Lutz MW, Saunders AM, et al. (2012) Longitudinal modeling of cognitive aging and the TOMM40 effect. Alzheimers Dement 8: 490-495.

81. Roses AD, Saunders AM, Huang Y, Strum J, Weisgraber $\mathrm{KH}$, et al. (2007) Complex disease-associated pharmacogenetics: drug efficacy, drug safety, and confirmation of a pathogenetic hypothesis (Alzheimer's disease). Pharmacogenomics J 7: 10-28. 